\title{
Supporting Information-1
}

\section{Ruthenium-Catalyzed [2+2] Cycloadditions of Alkynyl Sulfides and Alkynyl Sulfones}

\author{
Nicole Riddell and William Tam* \\ Guelph-Waterloo Centre for Graduate Work in Chemistry and Biochemistry, \\ Department of Chemistry, University of Guelph, Guelph, Ontario, Canada N1G 2W1
}

\section{Contents}

Part I: $\quad$ Preparation of Alkynyl Sulfides and Alkynyl Sulfones

p. S2-S8

Part II: Ru-catalyzed [2+2] Cycloadditions

p. S8- S18

Part III: Conversion of Cycloadduct 8c to Cycloadducts 25a-e

p. S18- S20

Part IV: ${ }^{1} \mathrm{H}$ and ${ }^{13} \mathrm{C}$ NMR Spectra of Alkynyl Sulfides \& Alkynyl Sulfones

p. S21- S33

Part V: $\quad{ }^{1} \mathrm{H}$ and ${ }^{13} \mathrm{C}$ NMR Spectra of Cycloadducts

p. S34- S58 
General Information: All reactions were carried out in an atmosphere of dry nitrogen at ambient temperature unless otherwise stated. Standard column chromatography was performed on 230-400 mesh silica gel using flash column chromatography techniques. ${ }^{1}$ All glassware was flame dried under an inert atmosphere of dry nitrogen. Chemical shifts for ${ }^{1} \mathrm{H}$ NMR spectra are reported in parts per million (ppm) from tetramethylsilane with the solvent resonance as the internal standard (chloroform: $\delta 7.26 \mathrm{ppm}$ ). Chemical shifts for ${ }^{13} \mathrm{C}$ NMR spectra are reported in parts per million (ppm) from tetramethylsilane with the solvent as the internal standard (deuterochloroform: $\delta 77.0 \mathrm{ppm}$ ).

Reagents: Unless stated otherwise, commercial reagents were used without purification. Solvents were purified by distillation under dry nitrogen: from $\mathrm{CaH}_{2}\left(\mathrm{CH}_{2} \mathrm{Cl}_{2}\right)$ and from potassium/benzophenone (THF). Bicyclic alkenes $\mathbf{9},{ }^{2} \mathbf{1 0},{ }^{3} \mathbf{1 1},{ }^{4} \mathbf{1 3}^{5}$ and the catalyst $\mathrm{Cp} * \mathrm{RuCl}(\mathrm{COD})^{6}$ were prepared according to literature procedures.

\section{Part I: Preparation of Alkynyl Sulfides and Alkynyl Sulfones}

General Procedure (A) for the Synthesis of the Alkynyl Sulfides. The terminal alkyne (1 eq) was dissolved in THF ( 0.45 M), under dry nitrogen, and cooled to $-78^{\circ} \mathrm{C}$ in a flame-dried round bottomed flask. A $2.5 \mathrm{M}$ solution of $n \mathrm{BuLi}$ was slowly added to the reaction flask and the mixture was stirred at $-78^{\circ} \mathrm{C}$ for 30 minutes. A solution of the disulfide ( $\left.1 \mathrm{eq}\right)$ in THF ( $\sim .55 \mathrm{M}$ ) was then added dropwise to the reaction via a cannula. The resulting solution was warmed to $25^{\circ} \mathrm{C}$ and subsequently stirred for $1 \mathrm{~h}$ 30 min. After cooling the reaction mixture to $-40^{\circ} \mathrm{C}$, a solution of $p$-nitrobenzyl bromide (1 eq) in THF ( $\sim 0.55 \mathrm{M})$ was added dropwise via a cannula. Upon warming to $25^{\circ} \mathrm{C}$, the mixture was stirred for another $1 \mathrm{~h} 30 \mathrm{~min}$ or until complete as monitored by TLC. The reaction was then quenched with saturated $\mathrm{NH}_{4} \mathrm{Cl}$ and the aqueous layer was extracted three times with diethyl ether. The organic layers were combined, dried over $\mathrm{MgSO}_{4}$, filtered, and concentrated using rotary evaporation. The resulting product was purified using flash chromatography (hexanes or ethyl acetate/hexanes mixture).

\footnotetext{
${ }^{1}$ W. C. Still, M. Kahn, A. Mitra, J. Org. Chem. 1978, 43, 2923.

${ }^{2}$ Michieletto, I.; Fabris, F.; De Lucchi, O. Tertrahedron (Asymmetry), 2000, 11, 2835.

${ }^{3}$ Story, P. R.; Fahrenholtz, S. R. Org. Synth. 1973, 151.

${ }^{4}$ Story, P. R.; Fahrenholtz, S. R. J. Org. Chem. 1963, 28, 1716.

${ }^{5}$ Nakayama, J.; Sakai, A.; Hoshimo, M. J. Org. Chem. 1984, 49, 5084.

${ }^{6}$ P. J. Fagan, W. S. Mahoney, J. C. Calabrese, I. D. Williams, Organometallics. 1990, 9, 1843.
} 


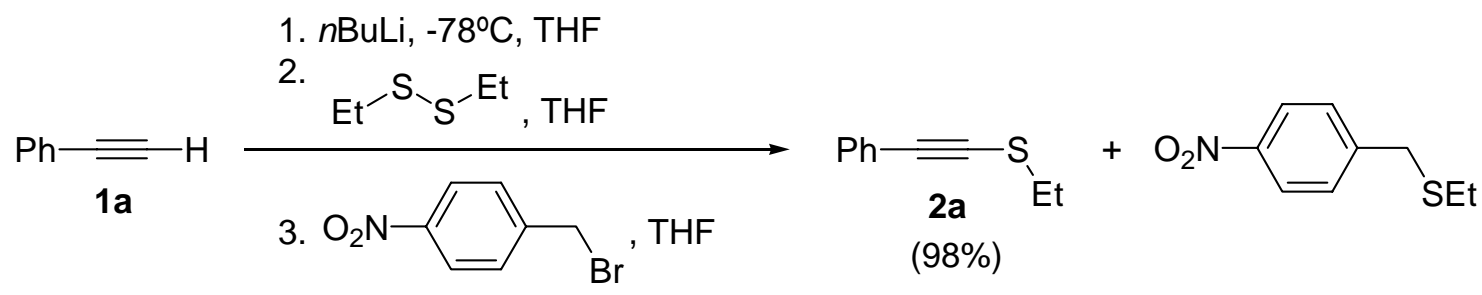

(2-Ethylsulfanylethynyl)benzene (2a). Following the above general procedure (A) with phenylacetylene 1a $(0.240 \mathrm{~mL}, 2.18 \mathrm{mmol})$ and $n \operatorname{BuLi}(2.5 \mathrm{M}$ in hexanes, $0.880 \mathrm{~mL}, 2.20 \mathrm{mmol})$, diethyl disulfide ( $0.240 \mathrm{~mL}, 1.95 \mathrm{mmol})$, and p-nitrobenzyl bromide (534.7 mg, $2.475 \mathrm{mmol})$. The crude product was purified by column chromatography (hexanes) to provide 2a (310.8 mg, 1.916 mmol, 98\%) as a colourless oil. $\mathrm{R}_{f} 0.49$ (EtOAc:hexanes=1:19); ${ }^{1} \mathrm{H}$ NMR $\left(\mathrm{CDCl}_{3}, 400 \mathrm{MHz}\right) \delta 7.41-$ 7.44 (m, 2H), 7.28-7.32 (m, 3H), 2.83 (q, 2H, $J=7.3 \mathrm{~Hz}), 1.47$ (t, 3H, $J=7.3 \mathrm{~Hz}) ;{ }^{13} \mathrm{C}$ NMR $\left(\mathrm{CDCl}_{3}\right.$, $100 \mathrm{MHz}) \delta 131.4,128.2,127.9,123.5,93.4,79.2,29.9,14.7$. The spectral data are identical to those reported in the literature. ${ }^{7}$

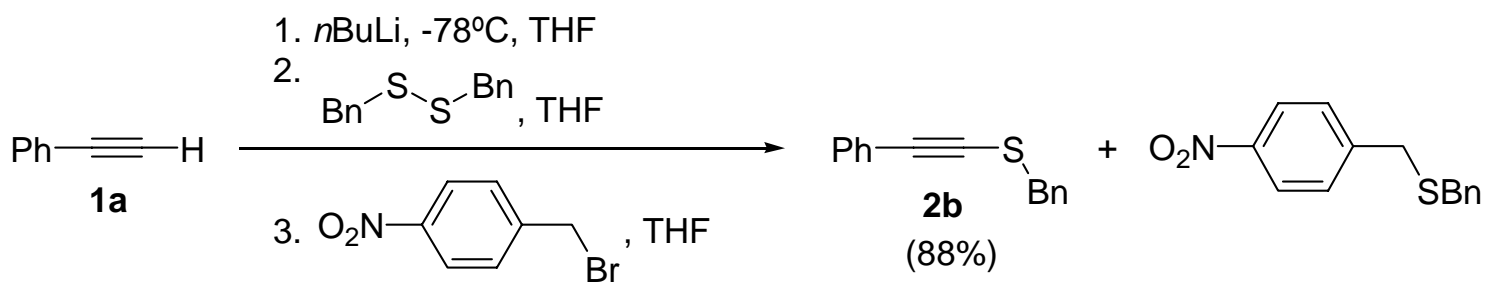

(2-Benzylsulfanylethynyl)benzene (2b). Following the above general procedure (A) with phenylacetylene 1a $(0.240 \mathrm{~mL}, 2.18 \mathrm{mmol})$ and $n \operatorname{BuLi}(2.5 \mathrm{M}$ in hexanes, $0.880 \mathrm{~mL}, 2.20 \mathrm{mmol})$, dibenzyl disulfide (540.9 mg, $2.195 \mathrm{mmol}$ ), and p-nitrobenzyl bromide (576.3 mg, $2.668 \mathrm{mmol}$ ). The crude product was purified by column chromatography (hexanes) to provide $\mathbf{2 b}$ (431.9 $\mathrm{mg}, 1.925$ mmol, 88\%) as a colourless oil. $\mathrm{R}_{f} 0.37$ (EtOAc:hexanes=1:19); ${ }^{1} \mathrm{H}$ NMR $\left(\mathrm{CDCl}_{3}, 400 \mathrm{MHz}\right) \delta 7.29-$ 7.42 (m, 10H), 4.03 (s, 2H); ${ }^{13} \mathrm{C} \mathrm{NMR}\left(\mathrm{CDCl}_{3}, 100 \mathrm{MHz}\right) \delta$ 136.5, 131.3, 129.1, 128.5, 128.2, 128.0, $127.7,123.3,94.6,79.1,40.4$. The spectral data are identical to those reported in the literature. ${ }^{8}$

\footnotetext{
${ }^{7}$ Voets, M.; Smet, M.; Dehaen, W. J. Chem. Soc., Perkin Trans. I 1999, 1473.

${ }^{8}$ Melandri, D.; Montevecchi, P.C.; Navacchia, M.L. Tetrahedron 1999, 55, 12227.
} 


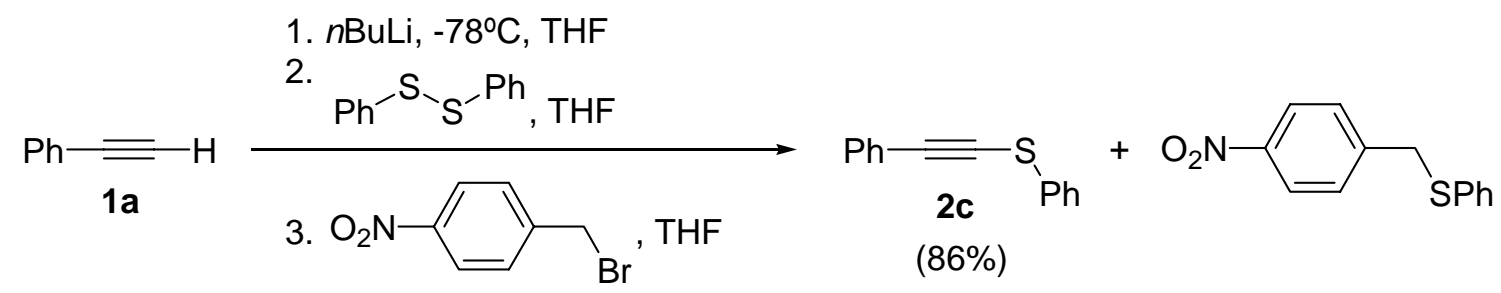

(2-Phenylsulfanylethynyl)benzene (2c). Following the above general procedure (A) with phenylacetylene 1a $(0.240 \mathrm{~mL}, 2.18 \mathrm{mmol})$ and $n \operatorname{BuLi}(2.5 \mathrm{M}$ in hexanes, $0.880 \mathrm{~mL}, 2.20 \mathrm{mmol})$, diphenyl disulfide (476.5 mg, $2.182 \mathrm{mmol}$ ), and p-nitrobenzyl bromide (576.8 mg, $2.669 \mathrm{mmol}$ ). The crude product was purified by column chromatography (hexanes) to provide 2c (394.9 mg, 1.878 mmol, 86\%) as a colourless oil. $\mathrm{R}_{f} 0.45$ (EtOAc:hexanes=1:19); ${ }^{1} \mathrm{H}$ NMR $\left(\mathrm{CDCl}_{3}, 400 \mathrm{MHz}\right) \delta 7.50$ 7.54 (m, 4H), 7.23-7.39 (m, 6H); ${ }^{13} \mathrm{C}$ NMR $\left(\mathrm{CDCl}_{3}, 75 \mathrm{MHz}\right) \delta$ 132.9, 131.7, 129.3, 128.6, 128.4, 126.5, 126.2, 122.9, 97.9, 75.5. The spectral data are identical to those reported in the literature. ${ }^{7}$

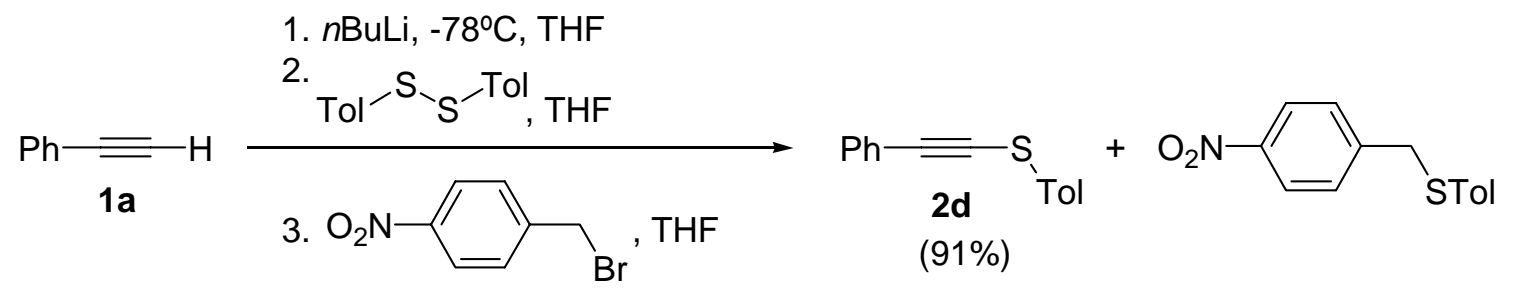

(2-p-Tolylsulfanylethynyl)benzene (2d). Following the above general procedure (A) with phenylacetylene 1a $(0.240 \mathrm{~mL}, 2.18 \mathrm{mmol})$ and $n \operatorname{BuLi}(2.5 \mathrm{M}$ in hexanes, $0.880 \mathrm{~mL}, 2.20 \mathrm{mmol})$, ditolyl disulfide (544.0 mg, $2.208 \mathrm{mmol}$ ), and p-nitrobenzyl bromide (468.2 mg, $2.167 \mathrm{mmol}$ ). The crude product was purified by column chromatography (hexanes) to provide 2d (445.2 mg, 1.985 mmol, 91\%) as a white solid. $\mathrm{R}_{f} 0.45$ (EtOAc:hexanes=1:19); ${ }^{1} \mathrm{H}$ NMR $\left(\mathrm{CDCl}_{3}, 400 \mathrm{MHz}\right) \delta 7.50$

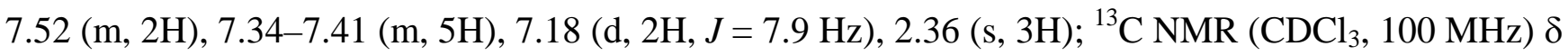
136.6, 131.7, 130.0, 129.1, 128.5, 128.3, 126.5, 123.0, 97.2, 76.1, 21.0. The spectral data are identical to those reported in the literature. ${ }^{9}$

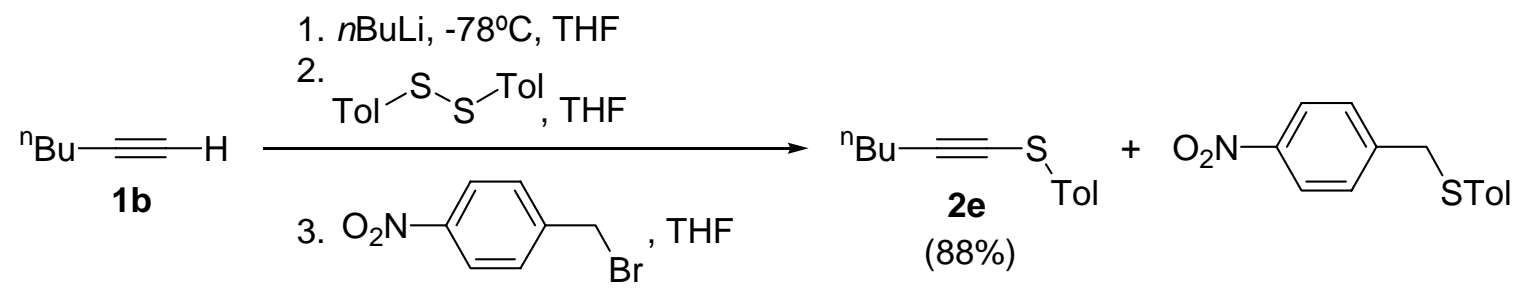

1-Hex-1-ynylsulfanyl-4-methylbenzene (2e). Following the above general procedure (A) with 1hexyne $1 \mathbf{b}(0.210 \mathrm{~mL}, 1.82 \mathrm{mmol})$ and $n \mathrm{BuLi}(2.5 \mathrm{M}$ in hexanes, $0.740 \mathrm{~mL}, 1.85 \mathrm{mmol})$, ditolyl

\footnotetext{
${ }^{9}$ Savarin, C.; Srogl, J.; Liebeskind, L.S. Org. Lett. 2001, 3, 91.
} 
disulfide (403.8 mg, $1.639 \mathrm{mmol}$ ), and p-nitrobenzyl bromide (427.6 mg, $1.979 \mathrm{mmol}$ ). The crude product was purified by column chromatography (hexanes) to provide 2e (293.5 mg, $1.436 \mathrm{mmol}$, 88\%) as a colourless oil. $\mathrm{R}_{f} 0.66$ (EtOAc:hexanes=3:7); ${ }^{1} \mathrm{H}$ NMR $\left(\mathrm{CDCl}_{3}, 400 \mathrm{MHz}\right) \delta 7.29-7.32(\mathrm{~m}$, 2H), 7.12-7.15 (m, 2H), 2.44 (t, 2H, $J=6.9 \mathrm{~Hz}), 2.33$ (s, 3H), 1.54-1.62 (m, 2H), 1.43-1.51 (m, 2H), 0.94 (t, 3H, $J=7.3 \mathrm{~Hz}$ ); ${ }^{13} \mathrm{C}$ NMR (APT, $\left.\mathrm{CDCl}_{3}, 100 \mathrm{MHz}\right) \delta 136.0,130.0,129.8,126.0,99.4,65.0$, 30.7, 22.0, 20.9, 20.0, 13.6. The spectral data are identical to those reported in the literature. ${ }^{10}$

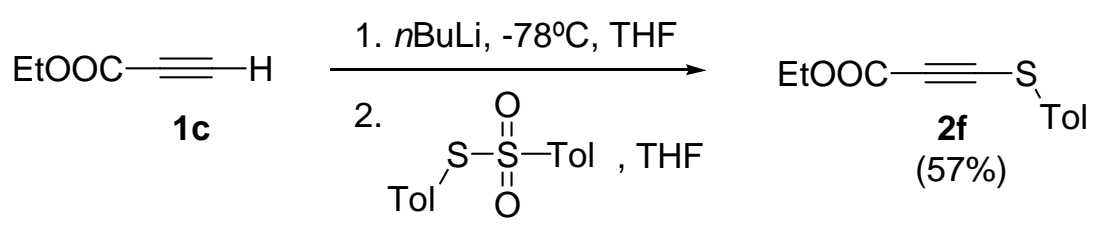

p-Tolylsulfanyl-propynoic acid ethyl ester (2f). To a cold solution $\left(-78^{\circ} \mathrm{C}\right)$ of ethyl propiolate 1c (0.170 mL, $1.68 \mathrm{mmol}, 1 \mathrm{eq})$ in THF under nitrogen was added $n \operatorname{BuLi}$ (2.5 M in hexanes, $0.660 \mathrm{~mL}$, $1.65 \mathrm{mmol}$ ) dropwise and the resulting mixture was stirred for 40 minutes. A solution of toluene-4thiosulfonic acid S-p-tolyl ester (457.5 mg, $1.643 \mathrm{mmol}, 1 \mathrm{eq}$ ) in THF was then added via a cannula and the mixture was stirred for an additional $1 \mathrm{~h}$. The reaction was quenched with water, and the aqueous layer was extracted three times with $\mathrm{CH}_{2} \mathrm{Cl}_{2}$. The organic layers were combined, dried over $\mathrm{MgSO}_{4}$, filtered, and concentrated using rotary evaporation. The crude product was purified by column chromatography (Et $t_{2} \mathrm{O}$ :hexanes=1:9) to provide $\mathbf{2 f}$ (206.8 $\left.\mathrm{mg}, 0.9388 \mathrm{mmol}, 57 \%\right)$ as a colourless oil. $\mathrm{R}_{f} 0.38\left(\mathrm{Et}_{2} \mathrm{O}\right.$ :hexanes=1:9); ${ }^{1} \mathrm{H} \mathrm{NMR}\left(\mathrm{CDCl}_{3}, 400 \mathrm{MHz}\right) \delta 7.36$ (d, 2H, $\left.J=8.2 \mathrm{~Hz}\right), 7.19$ (d, $2 \mathrm{H}, J=$ $8.0 \mathrm{~Hz}), 4.26$ (q, 2H, $J=7.1 \mathrm{~Hz}), 2.35$ (s, 3H), 1.32 (t, 3H, $J=7.1 \mathrm{~Hz}) ;{ }^{13} \mathrm{C} \mathrm{NMR}\left(\mathrm{CDCl}_{3}, 100 \mathrm{MHz}\right) \delta$ 152.9, 138.2, 130.4, 127.8, 125.6, 90.9, 80.6, 61.9, 21.0, 14.1. The spectral data are identical to those reported in the literature. ${ }^{11}$

General Procedure (B) for the synthesis of Alkynyl Sulfones. In a round bottom flask, the alkynyl sulfide (1 eq) was dissolved in $\mathrm{CH}_{2} \mathrm{Cl}_{2}(\sim 0.40 \mathrm{M})$ and cooled to $0^{\circ} \mathrm{C}$. A solution of $\mathrm{mCPBA}(2 \mathrm{eq})$ in $\mathrm{CH}_{2} \mathrm{Cl}_{2}(\sim 0.40 \mathrm{M})$ was then added slowly using a dropping funnel and the resulting mixture was allowed to warm to room temperature. After 1 hour, the reaction was gravity filtered and washed with sodium bicarbonate three times. The organic layer was then dried over $\mathrm{MgSO}_{4}$, filtered, and concentrated using rotary evaporation. The resulting crude product was purified using flash chromatography (hexanes or ethyl acetate/hexanes mixture).

\footnotetext{
${ }^{10}$ Zhao, S.H.; Samuel, O.; Kagan, H.B. Tetrahedron 1987, 43, 5135.

${ }^{11}$ Shen, M.; Schultz, A.G. Tetrahedron Lett. 1981, 22, 3347.
} 


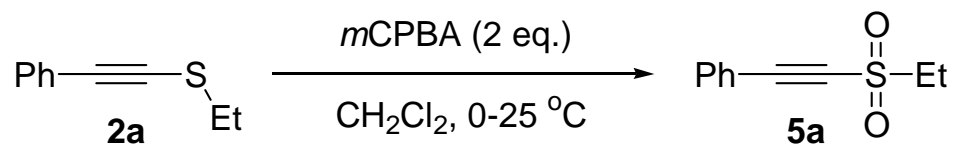

(82\%)

(2-Ethanesulfonylethynyl)benzene (5a). Following the above general procedure (B) with (2ethylsulfanylethynyl)benzene 2a (105.2 mg, $0.6484 \mathrm{mmol})$ and mCPBA (66.6\%, $327.6 \mathrm{mg}, 1.264$ mmol). The crude product was purified by column chromatography (EtOAc:hexanes=1:4) to provide 5a (103.3 mg, $0.5318 \mathrm{mmol}, 82 \%)$ as a colourless oil. $\mathrm{R}_{f} 0.38$ (EtOAc:hexanes=3:7); ${ }^{1} \mathrm{H}$ NMR $\left(\mathrm{CDCl}_{3}\right.$, $400 \mathrm{MHz}) \delta$ 7.48-7.57 (m, 3H), 7.38-7.42 (m, 2H), 3.28 (q, 2H, $J=7.4 \mathrm{~Hz}), 1.52$ (t, 3H, $J=7.4 \mathrm{~Hz}$ ); ${ }^{13} \mathrm{C} \mathrm{NMR}\left(\mathrm{APT}, \mathrm{CDCl}_{3}, 100 \mathrm{MHz}\right) \delta 132.7,131.6,128.7,117.4,92.4,82.5,52.7,7.6$. The spectral data are identical to those reported in the literature. ${ }^{12}$

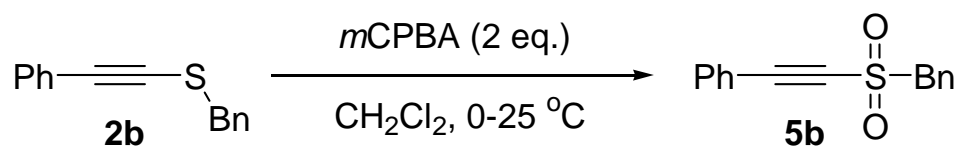

(52\%)

(2-Benzylsulfonylethynyl)benzene (5b). Following the above general procedure (B) with (2benzylsulfanylethynyl)benzene $2 \mathbf{b}$ (154.2 mg, $0.6874 \mathrm{mmol})$ and mCPBA (66.6\%, $512.7 \mathrm{mg}, 1.979$ mmol). The crude product was purified by column chromatography (EtOAc:hexanes=3:17) to provide $5 \mathbf{b}$ (80.5 mg, $0.314 \mathrm{mmol}, 52 \%$ ) as a white solid (m.p. $69-70^{\circ} \mathrm{C}$ ). $\mathrm{R}_{f} 0.42$ (EtOAc:hexanes=3:7); ${ }^{1} \mathrm{H}$ NMR $\left(\mathrm{CDCl}_{3}, 400 \mathrm{MHz}\right) \delta 7.37-7.52(\mathrm{~m}, 10 \mathrm{H}), 4.51$ (s, $\left.2 \mathrm{H}\right) ;{ }^{13} \mathrm{C} \mathrm{NMR}\left(\mathrm{CDCl}_{3}, 100 \mathrm{MHz}\right) \delta 132.7$, 131.7, 131.2, 129.4, 128.8, 128.7, 127.2, 117.4, 94.1, 82.5, 64.5. The spectral data are identical to those reported in the literature. ${ }^{13}$

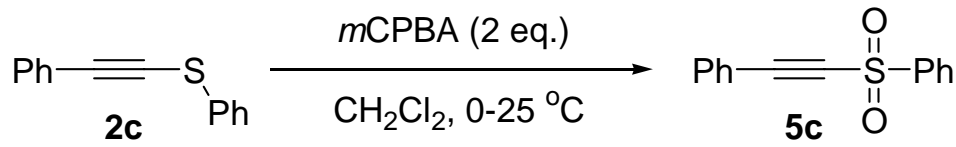

(37\%)

(2-Phenylsulfonylethynyl)benzene (5c). Following the above general procedure (B) with (2phenylsulfanylethynyl)benzene 2c (105.9 mg, $0.5036 \mathrm{mmol})$ and mCPBA (66.6\%, $275.8 \mathrm{mg}, 1.064$ mmol). The crude product was purified by column chromatography (EtOAc:hexanes=1:9) to provide 5c (45.3 mg, $0.187 \mathrm{mmol}, 37 \%$ ) as a yellow solid (m.p. $62-63^{\circ} \mathrm{C}$ ). $\mathrm{R}_{f} 0.41$ (EtOAc:hexanes=3:7); ${ }^{1} \mathrm{H}$ NMR $\left(\mathrm{CDCl}_{3}, 400 \mathrm{MHz}\right) \delta 8.07-8.10(\mathrm{~m}, 2 \mathrm{H}), 7.35-7.72(\mathrm{~m}, 8 \mathrm{H}) ;{ }^{13} \mathrm{C} \mathrm{NMR}\left(\mathrm{CDCl}_{3}, 100 \mathrm{MHz}\right) \delta$

\footnotetext{
12 Truce, W.E.; Wolf, G.C. J. Org. Chem. 1971, 36, 1727.

${ }^{13}$ Ziegler, G.R.; Welch, C.A.; Orzech, C.E.; Kikkawa, S.; Miller, S. J. Am. Chem. Soc. 1963, 85, 1648.
} 
141.8, 134.2, 132.7, 131.6, 129.4, 128.7, 127.4, 117.8, 93.5, 85.3. The spectral data are identical to those reported in the literature. ${ }^{14}$

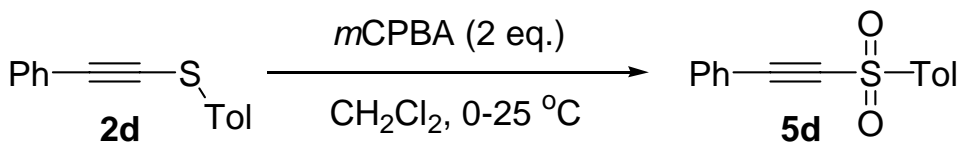

(82\%)

(2-p-Tolylsulfonylethynyl)benzene (5d). Following the above general procedure (B) with (2-ptolylsulfanylethynyl)benzene 2d (200.0 mg, $0.8916 \mathrm{mmol})$ and mCPBA (66.6\%, $570.5 \mathrm{mg}, 2.182$ mmol). The crude product was purified by column chromatography (EtOAc:hexanes=1:4) to provide 5d (188.1 mg, $0.7338 \mathrm{mmol}, 82 \%)$ as a white solid (m.p. $\left.73-75^{\circ} \mathrm{C}\right) . \mathrm{R}_{f} 0.52$ (EtOAc:hexanes=2:3); ${ }^{1} \mathrm{H}$ NMR $\left(\mathrm{CDCl}_{3}, 400 \mathrm{MHz}\right) \delta 7.96(\mathrm{~d}, 2 \mathrm{H}, J=8.4 \mathrm{~Hz}), 7.35-7.53(\mathrm{~m}, 7 \mathrm{H}), 2.47$ (s, 3H); ${ }^{13} \mathrm{C}$ NMR $\left(\mathrm{CDCl}_{3}, 100 \mathrm{MHz}\right) \delta 145.4,138.9,132.7,131.4,130.0,128.6,127.5,117.9,92.9,85.5,21.7$. The spectral data are identical to those reported in the literature. ${ }^{15}$

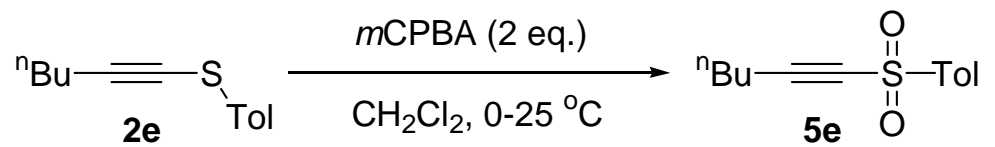

$(89 \%)$

1-(Hex-1-yne-1-sulfonyl)-4-methylbenzene (5e). Following the above general procedure (B) with 1hex-1-ynylsulfanyl-4-methylbenzene 2e (109.1 mg, $0.5339 \mathrm{mmol})$ and mCPBA (66.6\%, $285.8 \mathrm{mg}$, $1.103 \mathrm{mmol}$ ). The crude product was purified by column chromatography (EtOAc:hexanes=1:9) to provide 5e (111.9 mg, $0.4735 \mathrm{mmol}, 89 \%)$ as a colourless oil. $\mathrm{R}_{f} 0.49$ (EtOAc:hexanes=3:7); ${ }^{1} \mathrm{H}$ NMR $\left(\mathrm{CDCl}_{3}, 400 \mathrm{MHz}\right) \delta 7.87(\mathrm{~d}, 2 \mathrm{H}, J=8.3 \mathrm{~Hz}), 7.36$ (d, 2H, $\left.J=8.3 \mathrm{~Hz}\right), 2.46(\mathrm{~s}, 3 \mathrm{H}), 2.35$ (t, $2 \mathrm{H}, J=$ 7.1 Hz), 1.49-1.56 (m, 2H), 1.33-1.39 (m, 2H), 0.88 (t, 3H, $J=7.3 \mathrm{~Hz}) ;{ }^{13} \mathrm{C}$ NMR (APT, $\mathrm{CDCl}_{3}, 100$ MHz) $\delta$ 145.0, 139.2, 129.8, 127.2, 97.3, 78.3, 28.9, 21.9, 21.7, 18.6, 13.4. HRMS calcd. For $\mathrm{C}_{13} \mathrm{H}_{16} \mathrm{SO}_{2}: \mathrm{m} / \mathrm{z} 236.0871$, found $\mathrm{m} / \mathrm{z}$ 236.0876. The spectral data are identical to those reported in the literature. $^{16}$

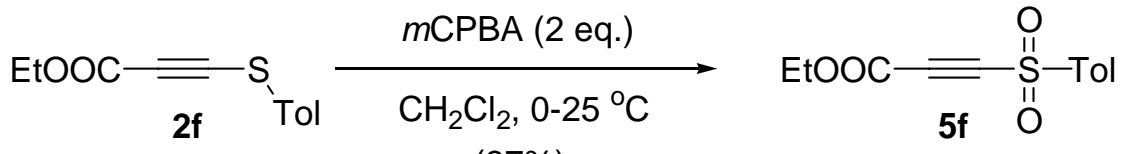

(37\%)

(Toluene-4-sulfonyl)-propynoic acid ethyl ester (5f). Following the above general procedure (B) with p-tolylsulfanyl-propynoic acid ethyl ester 2 f (107.2 mg, $0.4867 \mathrm{mmol})$ and mCPBA (66.6\%, 238.6

\footnotetext{
${ }^{14}$ Padwa, A.; Wannamaker, M.W. Tetrahedron 1990, 46, 1145.

${ }^{15}$ Qian, H.; Huang, X. Tetrahedron Lett. 2002, 43, 1059.
} 
$\mathrm{mg}, 0.9208 \mathrm{mmol}$ ). The crude product was purified by column chromatography (EtOAc:hexanes=1:9) to provide $5 \mathbf{f}$ (46.0 mg, $0.182 \mathrm{mmol}$, 37\%) as a colourless oil. $\mathrm{R}_{f} 0.44$ (EtOAc:hexanes=3:7); ${ }^{1} \mathrm{H}$ NMR $\left(\mathrm{CDCl}_{3}, 400 \mathrm{MHz}\right) \delta$ 7.88-7.91 (m, 2H), 7.40-7.42 (m, 2H), 4.27 (q, 2H, $\left.J=7.1 \mathrm{~Hz}\right), 2.48$ (s, 3H), 1.30 (t, 3H, $J=7.1 \mathrm{~Hz}) ;{ }^{13} \mathrm{C}$ NMR (APT, $\left.\mathrm{CDCl}_{3}, 100 \mathrm{MHz}\right) \delta 151.0,146.8,136.8,130.3,128.1,79.4,79.3$, $63.5,21.8,13.8$. The spectral data are identical to those reported in the literature. ${ }^{11}$

\section{Part II: Ru-catalyzed [2+2] cycloadditions}

General Procedure (C) for the Ru-Catalyzed [2+2] Cycloadditions. A mixture of bicyclic alkene (2.5-5 eq), alkynyl sulfur substrate (1 eq) and THF (0.2-0.5 M with respect to the sulfur substrate) was prepared in an oven-dried vial. The contents of this vial were then transferred via a cannula to an ovendried screw-cap vial containing $\mathrm{Cp} * \operatorname{RuCl}(\mathrm{COD})$ (weighed out in a dry box, 5-10 mol\%) under nitrogen. The reaction mixture was stirred in the dark at $90^{\circ} \mathrm{C}$ for $72-168 \mathrm{~h}$. The crude product was purified by flash chromatography to give the corresponding cycloadduct (hexanes or ethyl acetate/hexanes mixture).

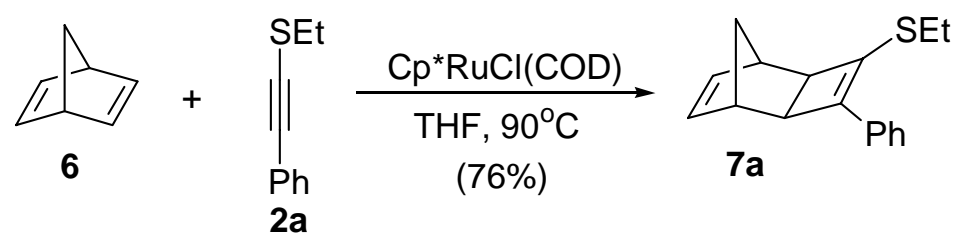

Cycloadduct 7a (Table 1, entry 1). Following the above general procedure (C) with norbornadiene 6 (65.0 $\mu \mathrm{L}, 0.603 \mathrm{mmol}$ ), alkynyl sulfide $2 \mathbf{a}(29.9 \mathrm{mg}, 0.184 \mathrm{mmol})$, THF (0.3 mL), and Cp*RuCl(COD) (7.4 mg, $0.020 \mathrm{mmol}$ ). The reaction mixture was stirred in the dark at $90{ }^{\circ} \mathrm{C}$ for $168 \mathrm{~h}$. The crude product was purified by column chromatography (hexanes) to provide cycloadduct 7a (35.5 mg, 0.140 mmol, 76\%) as a colourless oil. $\mathrm{R}_{\mathrm{f}} 0.54$ (EtOAc:hexanes= 1:9); IR $\left(\mathrm{CH}_{2} \mathrm{Cl}_{2}, \mathrm{NaCl}\right) 3056$ (m), 3024 (w), 2969 (s), 2928 (s), 2871 (w), 1603 (s), 1488 (s), 1446 (s), 1263 (s), 1191 (s), 775 (s), 762 (s), 691 (s) $\mathrm{cm}^{-1}$; ${ }^{1} \mathrm{H}$ NMR $\left(\mathrm{CDCl}_{3}, 400 \mathrm{MHz}\right)$ 8 7.43-7.46 (m, 2H), 7.29-7.38 (m, 2H), 7.20 (m, 1H), 6.24 (dd, 1H, $J=3.1,5.5 \mathrm{~Hz}$ ), 6.18 (dd, 1H, $J=3.1,5.5 \mathrm{~Hz}), 2.82-3.00$ (m, 2H), 2.73 (s, 1H), 2.73 (d, 1H, $J=$ $2.4 \mathrm{~Hz}$ ), 2.69 (m, 1H), 2.63 (br s, 1H), 1.48 (m, 2H), 1.38 (t, 3H, $J=7.4 \mathrm{~Hz}$ ); ${ }^{13} \mathrm{C}$ NMR (APT, $\mathrm{CDCl}_{3}$, $100 \mathrm{MHz}$ ) $\delta$ 139.7, 136.6, 135.3, 135.1, 134.1, 128.3, 126.5, 125.7, 46.1, 43.3, 40.4, 39.8, 39.7, 25.6,

\footnotetext{
${ }^{16}$ Melandri, D.; Montevecchi, P.C.; Navacchia, M.L. Tetrahedron 1999, 55, 12227.
} 
16.1. HRMS calcd. For $\mathrm{C}_{17} \mathrm{H}_{18} \mathrm{~S}$ : $\mathrm{m} / \mathrm{z}$ 254.1129, found $\mathrm{m} / \mathrm{z}$ 254.1134. Anal. calcd. For $\mathrm{C}_{17} \mathrm{H}_{18} \mathrm{~S}$ : C, 80.26; H, 7.13. Found C, 80.10; H, 7.46.

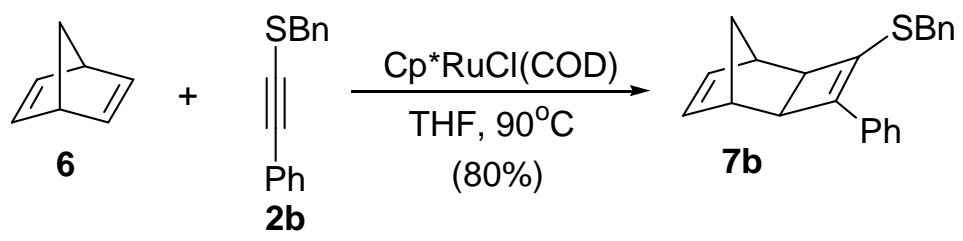

Cycloadduct 7b (Table 1, entry 2). Following the above general procedure (C) with norbornadiene 6 (60.0 $\mu \mathrm{L}, 0.556 \mathrm{mmol}$ ), alkynyl sulfide $2 \mathbf{b}$ (27.5 mg, $0.123 \mathrm{mmol}$ ), THF (0.3 mL), and Cp*RuCl(COD) (6.8 mg, $0.018 \mathrm{mmol}$ ). The reaction mixture was stirred in the dark at $90{ }^{\circ} \mathrm{C}$ for $168 \mathrm{~h}$. The crude product was purified by column chromatography (hexanes) to provide cycloadduct $7 \mathbf{b}$ (31.1 mg, 0.0983 mmol, 80\%) as a slightly yellow oil. $\mathrm{R}_{\mathrm{f}} 0.56$ (EtOAc:hexanes= 1:9); IR $\left(\mathrm{CH}_{2} \mathrm{Cl}_{2}, \mathrm{NaCl}\right) 3058$ (w), 3028 (w), 2967 (w), 1770 (w), 1601 (m), 1489 (m), 1320 (m), 1191 (m), 1070 (w), 950 (w), 693 (s) $\mathrm{cm}^{-1}$; ${ }^{1} \mathrm{H}$ NMR $\left(\mathrm{CDCl}_{3}, 400 \mathrm{MHz}\right) \delta$ 7.40-7.44 (m, 4H), 7.31-7.36 (m, 4H), 7.18-7.28 (m, 2H), 6.21 (dd, 1H, $J=3.1,5.6 \mathrm{~Hz}$ ), 6.15 (dd, 1H, $J=3.1,5.6 \mathrm{~Hz}), 4.14$ (ABq, 2H, $J=13.2 \mathrm{~Hz}), 2.71-2.72$ (m, 2H), 2.65 (d, 1H, $J=3.8 \mathrm{~Hz}$ ), 2.53 (d, 1H, $J=1.2 \mathrm{~Hz}$ ), 1.48 (d, 1H, $J=9.1 \mathrm{~Hz}), 1.37$ (d, $1 \mathrm{H}, J=9.1$ $\mathrm{Hz}) ;{ }^{13} \mathrm{C} \mathrm{NMR}\left(\mathrm{APT}, \mathrm{CDCl}_{3}, 100 \mathrm{MHz}\right) \delta$ 140.0, 137.8, 136.5, 135.4, 134.7, 134.0, 128.7, 128.6, 128.4, 127.3, 126.6, 125.8, 46.5, 43.5, 40.3, 39.9, 39.6, 35.9. HRMS calcd. For $\mathrm{C}_{22} \mathrm{H}_{20} \mathrm{~S}$ : m/z 316.1286, found $\mathrm{m} / \mathrm{z} 316.1290$.

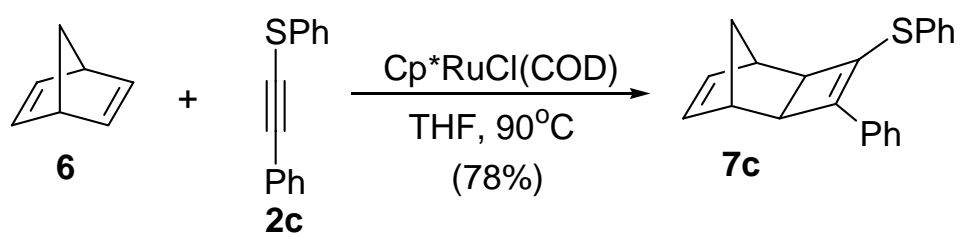

Cycloadduct 7c (Table 1, entry 3). Following the above general procedure (C) with norbornadiene 6 (50.0 $\mu \mathrm{L}, 0.463 \mathrm{mmol}$ ), alkynyl sulfide 2c (32.3 mg, $0.154 \mathrm{mmol})$, THF (0.3 mL), and Cp*RuCl(COD) (6.2 mg, $0.016 \mathrm{mmol}$ ). The reaction mixture was stirred in the dark at $90{ }^{\circ} \mathrm{C}$ for $168 \mathrm{~h}$. The crude product was purified by column chromatography (hexanes) to provide cycloadduct 7c (36.3 mg, 0.120 mmol, 78\%) as a white solid (m.p. $\left.50-52^{\circ} \mathrm{C}\right) . \quad \mathrm{R}_{\mathrm{f}} 0.56$ (EtOAc:hexanes= 1:9); IR $\left(\mathrm{CH}_{2} \mathrm{Cl}_{2}, \mathrm{NaCl}\right)$ 3057 (m), 3022 (w), 2969 (s), 2944 (m), 2873 (w), 1603 (m), 1582 (m), 1476 (m), 1446 (m), 1320 (s), 1287 (m), 1190 (m), 949 (m), 749 (m), 691 (s) cm ${ }^{-1} ;{ }^{1} \mathrm{H}$ NMR $\left(\mathrm{CDCl}_{3}, 400 \mathrm{MHz}\right) \delta$ 7.44-7.47 (m, 4H), 7.22-7.32 (m, 5H), 7.17 (m, 1H), 6.07 (m, 1H), 5.89 (m, 1H), 2.64 (d, 1H, J = 3.8Hz), 2.62 (br s, 1H), 2.43 (d, 1H, $J=3.8 \mathrm{~Hz}$ ), 2.03 (br s, $1 \mathrm{H}), 1.39$ (d, $1 \mathrm{H}, J=9.1 \mathrm{~Hz}$ ), 1.23 (d, $1 \mathrm{H}, J=9.1 \mathrm{~Hz}$ ); ${ }^{13} \mathrm{C}$ NMR 
(APT, $\left.\mathrm{CDCl}_{3}, 100 \mathrm{MHz}\right) \delta 142.6,136.3,135.5,133.7,133.5,133.1,132.0,129.0,128.4,127.9,127.1$, 125.9, 46.8, 43.7, 40.2, 39.8, 38.9. HRMS calcd. For $\mathrm{C}_{21} \mathrm{H}_{18} \mathrm{~S}$ : m/z 302.1129, found m/z 302.1133. Anal. calcd. For $\mathrm{C}_{21} \mathrm{H}_{18} \mathrm{~S}$ : C, 83.40; H, 6.00. Found C, 83.56; H, 5.82.

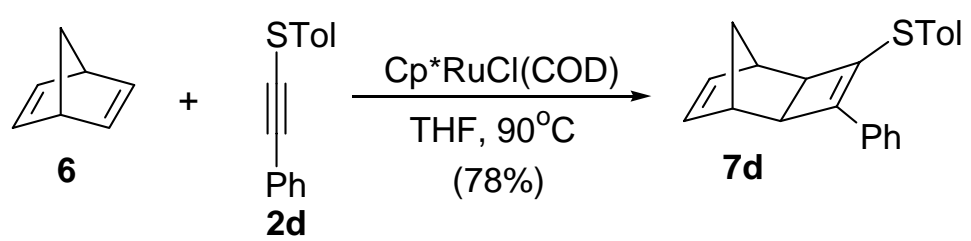

Cycloadduct 7d (Table 1, entry 4). Following the above general procedure (C) with norbornadiene 6 (50.0 $\mathrm{L}, 0.463 \mathrm{mmol}$ ), alkynyl sulfide $2 \mathbf{d}$ (35.3 mg, $0.157 \mathrm{mmol}$ ), THF (0.3 mL), and Cp*RuCl(COD) (5.6 mg, $0.015 \mathrm{mmol}$ ). The reaction mixture was stirred in the dark at $90{ }^{\circ} \mathrm{C}$ for $168 \mathrm{~h}$. The crude product was purified by column chromatography (hexanes) to provide cycloadduct $\mathbf{7 d}$ ( $38.9 \mathrm{mg}, 0.123$ mmol, 78\%) as a white solid (m.p. $\left.40-42^{\circ} \mathrm{C}\right) . \mathrm{R}_{\mathrm{f}} 0.66$ (EtOAc:hexanes= 1:4); IR $\left(\mathrm{CH}_{2} \mathrm{Cl}_{2}, \mathrm{NaCl}\right.$ ) 3056 (w), 3021 (w), 2970 (m), 2945 (m), 2872 (w), 1603 (m), 1490 (m), 1446 (m), 1320 (m), 1190 (m), 948 (m), 810 (m), 710 (s) $\mathrm{cm}^{-1}$; ${ }^{1} \mathrm{H}$ NMR $\left(\mathrm{CDCl}_{3}, 400 \mathrm{MHz}\right) \delta 7.53$ (m, 2H), 7.37-7.51 (m, 4H), 7.23 (m, 1H), 7.17 (d, 2H, $J=8.0 \mathrm{~Hz}$ ), 6.15 (m, 1H), 5.97 (m, 1H), 2.70 (s, 1H), 2.69 (s, 1H), 2.47 (d, 1H, $J$ = $3.7 \mathrm{~Hz}$ ), 2.38 (s, 3H), 2.09 (br s, 1H), 1.47 (d, $1 \mathrm{H}, J=9.1 \mathrm{~Hz}), 1.30$ (d, $1 \mathrm{H}, J=9.2 \mathrm{~Hz}$ ); ${ }^{13} \mathrm{C}$ NMR $\left(\mathrm{CDCl}_{3}, 100 \mathrm{MHz}\right) \delta 141.4,138.1,136.3,135.5,134.2,133.9,133.5,129.8,128.4,128.1,126.9,125.9$, 46.7, 43.5, 40.2, 39.8, 38.9, 21.2. HRMS calcd. For $\mathrm{C}_{22} \mathrm{H}_{20} \mathrm{~S}$ : m/z 316.1286, found m/z 316.1291.

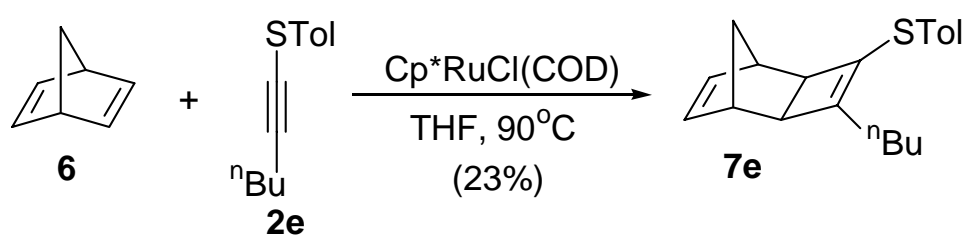

Cycloadduct 7e (Table 1, entry 5). Following the above general procedure (C) with norbornadiene 6 (65.0 $\mu \mathrm{L}, 0.603 \mathrm{mmol})$, alkynyl sulfide $2 \mathbf{e}(28.4 \mathrm{mg}, 0.139 \mathrm{mmol})$, THF $(0.3 \mathrm{~mL})$, and $\mathrm{Cp} * \operatorname{RuCl}(\mathrm{COD})$ (6.8 mg, $0.018 \mathrm{mmol})$. The reaction mixture was stirred in the dark at $90{ }^{\circ} \mathrm{C}$ for $168 \mathrm{~h}$. The crude product was purified by column chromatography (hexanes) to provide cycloadduct 7 e $(9.5 \mathrm{mg}, 0.032$ mmol, 23\%) as a colourless oil. $\mathrm{R}_{\mathrm{f}} 0.62$ (EtOAc:hexanes= 1:9); IR $\left(\mathrm{CH}_{2} \mathrm{Cl}_{2}, \mathrm{NaCl}\right) 3126$ (w), 3058 (w), 2959 (s), 2928 (s), 2871 (m), 1894 (w), 1624 (w), 1492 (s), 1449 (m), 1319 (m), 1200 (m), 1086 (m), 807 (s) cm ${ }^{-1} ;{ }^{1} \mathrm{H}$ NMR (CDCl, $\left.400 \mathrm{MHz}\right) \delta 7.30$ (d, 2H, $J=8.1 \mathrm{~Hz}$ ), 7.10 (d, 2H, $J=7.9 \mathrm{~Hz}$ ), 6.08 (dd, $1 \mathrm{H}, J=5.5,3.0 \mathrm{~Hz}$ ), 5.97 (dd, $1 \mathrm{H}, J=5.5,3.0 \mathrm{~Hz}$ ), 2.46 (br s, 1H), 2.38 (br s, $1 \mathrm{H}$ ), 2.35 (s, 1H), 2.33 (s, 3H), 2.22 (br s, 1H), 2.09-2.15 (m, 2H), 1.41-1.49 (m, 3H), 1.29-1.38 (m, 3H), 0.91 (t, 3H, 
$J=7.3 \mathrm{~Hz}) ;{ }^{13} \mathrm{C} \mathrm{NMR}\left(\mathrm{CDCl}_{3}, 100 \mathrm{MHz}\right) \delta 137.2,136.5,136.0,132.1,131.5,130.6,130.0,47.5,46.4$, 40.5, 39.7, 39.0, 29.8, 28.0, 23.3, 21.5, 14.3. HRMS calcd. For $\mathrm{C}_{20} \mathrm{H}_{24} \mathrm{~S}$ : $\mathrm{m} / \mathrm{z}$ 296.1599, found $\mathrm{m} / \mathrm{z}$ 296.1593.

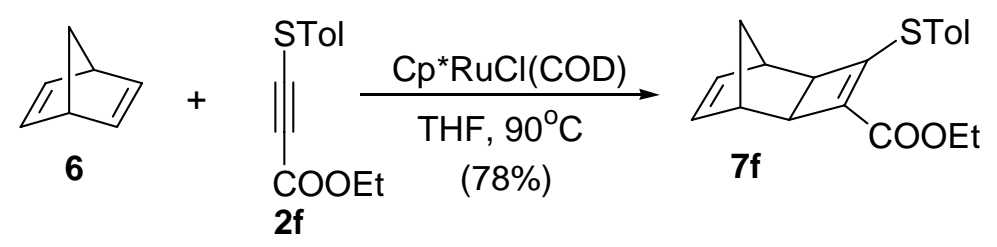

Cycloadduct 7f (Table 1, entry 6). Following the above general procedure (C) with norbornadiene 6 (45.0 $\mu \mathrm{L}, 0.417 \mathrm{mmol})$, alkynyl sulfide $2 \mathbf{f}(27.8 \mathrm{mg}, 0.126 \mathrm{mmol})$, THF (0.3 mL), and Cp*RuCl(COD) (6.6 mg, $0.017 \mathrm{mmol}$ ). The reaction mixture was stirred in the dark at $90{ }^{\circ} \mathrm{C}$ for $168 \mathrm{~h}$. The crude product was purified by column chromatography (EtOAc:hexanes=1:9) to provide cycloadduct $\mathbf{7 f}$ (30.8 mg, $0.0986 \mathrm{mmol}, 78 \%)$ as a slightly yellow oil. $\mathrm{R}_{\mathrm{f}} 0.39$ (EtOAc:hexanes= 1:4); $\mathrm{IR}\left(\mathrm{CH}_{2} \mathrm{Cl}_{2}, \mathrm{NaCl}\right)$ 3058 (w), 2977 (m), 2948 (m), 2873 (w), 1700 (s), 1579 (m), 1493 (m), 1452 (w), 1366 (w), 1314 (m), 1285 (m), 1193 (s), 1106 (m), 1035 (m), 812 (m), 713 (m) cm ${ }^{-1} ;{ }^{1} \mathrm{H}^{\mathrm{NMR}}\left(\mathrm{CDCl}_{3}, 400 \mathrm{MHz}\right) \delta 7.42$ (d, $2 \mathrm{H}, J=8.1 \mathrm{~Hz}$ ), 7.19 (d, 2H, $J=8.1 \mathrm{~Hz}$ ), 6.10 (dd, $1 \mathrm{H}, J=3.1,2.8 \mathrm{~Hz}$ ), 5.86 (dd, $1 \mathrm{H}, J=3.1,2.8 \mathrm{~Hz}$ ), 4.24 (q, 2H, $J=6.4 \mathrm{~Hz}$ ), 2.67 (br s, 1H), 2.54 (d, 1H, $J=3.7 \mathrm{~Hz}$ ), 2.39 (s, 3H), 2.34 (d, 1H, $J=3.7$ Hz), 1.89 (br s, 1H), 1.40 (d, 1H, $J=9.3 \mathrm{~Hz}$ ), 1.34 (t, 3H, $J=7.1 \mathrm{~Hz}$ ), 1.28 (d, $1 \mathrm{H}, J=9.3 \mathrm{~Hz}$ ); ${ }^{13} \mathrm{C}$ NMR (APT, $\left.\mathrm{CDCl}_{3}, 100 \mathrm{MHz}\right) \delta 162.8,158.0,139.4,136.9,134.8,134.6,129.9,128.8,125.9,56.9$, 47.3, 43.2, 39.9, 39.5, 38.5, 21.3, 14.5. HRMS calcd. For $\mathrm{C}_{19} \mathrm{H}_{20} \mathrm{SO}_{2}: \mathrm{m} / \mathrm{z} 312.1184$, found $\mathrm{m} / \mathrm{z}$ 312.1189.

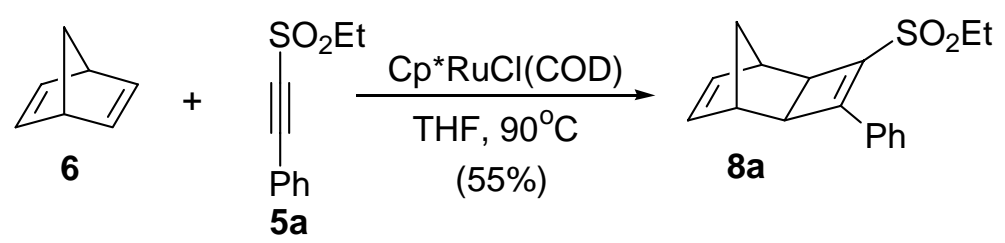

Cycloadduct 8a (Table 1, entry 7). Following the above general procedure (C) with norbornadiene 6 (50.0 $\mu \mathrm{L}, 0.463 \mathrm{mmol})$, alkynyl sulfone $5 \mathbf{a}(31.3 \mathrm{mg}, 0.161 \mathrm{mmol})$, THF $(0.3 \mathrm{~mL})$, and $\mathrm{Cp} * \mathrm{RuCl}(\mathrm{COD})(5.9 \mathrm{mg}, 0.016 \mathrm{mmol})$. The reaction mixture was stirred in the dark at $90^{\circ} \mathrm{C}$ for $72 \mathrm{~h}$. The crude product was purified by column chromatography (EtOAc:hexanes $=1: 9$ ) to provide cycloadduct 8a (25.3 mg, $0.0883 \mathrm{mmol}, 55 \%$ ) as a slightly yellow solid (m.p. $59-61^{\circ} \mathrm{C}$ ). $\mathrm{R}_{\mathrm{f}} 0.43$ (EtOAc:hexanes= 3:7); IR ( $\left.\mathrm{CH}_{2} \mathrm{Cl}_{2}, \mathrm{NaCl}\right) 3062$ (w), 2975 (m), 2944 (m), 2878 (w), 1608 (m), 1490 (m), 1449 (m), 1304 (s), 1142 (s), 1116 (s), 779 (s), 709 (s), 693 (m) cm ${ }^{-1} ;{ }^{1} \mathrm{H}$ NMR ( $\mathrm{CDCl}_{3}, 400 \mathrm{MHz}$ ) 
א 7.91-7.95 (m, 2H), 7.41-7.44 (m, 3H), 6.22-6.25 (m, 2H), 3.09 (q, 2H, J = 7.5 Hz), 2.89 (br s, 1H), 2.82 (d, 1H, $J=3.7 \mathrm{~Hz}$ ), 2.79 (br s, 1H), 2.73 (d, 1H, $J=3.7 \mathrm{~Hz}), 1.54$ (d, 1H, J = 9.6 Hz), 1.47 (d, $1 \mathrm{H}$, $J=9.6 \mathrm{~Hz}), 1.38$ (t, 3H, $J=7.5 \mathrm{~Hz}) ;{ }^{13} \mathrm{C} \mathrm{NMR}\left(\mathrm{APT}, \mathrm{CDCl}_{3}, 100 \mathrm{MHz}\right) \delta 157.4,136.1,135.6,133.7$, 130.9, 130.6, 129.1, 128.8, 48.9, 45.0, 43.6, 39.6, 39.3, 39.0, 6.6. HRMS calcd. For $\mathrm{C}_{17} \mathrm{H}_{18} \mathrm{SO}_{2}$ : m/z 286.1028, found $\mathrm{m} / \mathrm{z} 286.1022$.

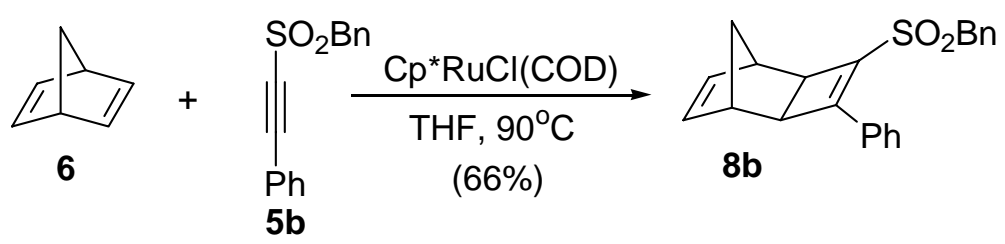

Cycloadduct 8b (Table 1, entry 8). Following the above general procedure (C) with norbornadiene 6 (40.0 $\mu \mathrm{L}, 0.371 \mathrm{mmol}$ ), alkynyl sulfone $5 \mathbf{b}$ (30.9 mg, $0.1206 \mathrm{mmol})$, THF (0.3 mL), and Cp*RuCl(COD) (6.1 mg, $0.016 \mathrm{mmol}$ ). The reaction mixture was stirred in the dark at $90{ }^{\circ} \mathrm{C}$ for $72 \mathrm{~h}$. The crude product was purified by column chromatography (EtOAc:hexanes= 1:19) to provide cycloadduct $\mathbf{8 b}$ (27.7 mg, $0.0795 \mathrm{mmol}, 66 \%$ ) as a white solid (m.p. 127 - 130 ${ }^{\circ} \mathrm{C}$ ). $\mathrm{R}_{\mathrm{f}} 0.52$ (EtOAc:hexanes= 3:7); IR ( $\left.\mathrm{CH}_{2} \mathrm{Cl}_{2}, \mathrm{NaCl}\right) 3061$ (w), 3032 (w), 2975 (m), 2949 (m), 1607 (m), 1490

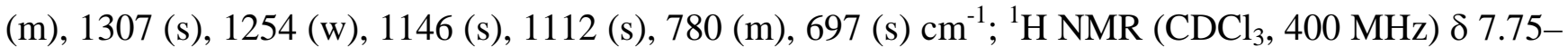
7.77 (m, 2H), 7.28-7.41 (m, 8H), 6.15-6.19 (m, 2H), 4.31 (s, 2H), 2.76 (br s, 1H), 2.72 (s, 1H), 2.71 (s, 1H), 2.40 (d, 1H, $J=3.4 \mathrm{~Hz}$ ), 1.47 (d, $1 \mathrm{H}, J=9.7 \mathrm{~Hz}$ ), 1.39 (d, $1 \mathrm{H}, J=9.7 \mathrm{~Hz}$ ); ${ }^{13} \mathrm{C}$ NMR (APT, $\left.\mathrm{CDCl}_{3}, 100 \mathrm{MHz}\right) \delta 158.4,136.3,135.4,133.3,130.9,130.9,130.5,128.9,128.8,128.7,128.6,127.6$, 61.1, 45.4, 43.4, 39.6, 39.1, 39.1. HRMS calcd. For $\mathrm{C}_{22} \mathrm{H}_{20} \mathrm{SO}_{2}: \mathrm{m} / \mathrm{z}$ 348.1184, found m/z 348.1178.

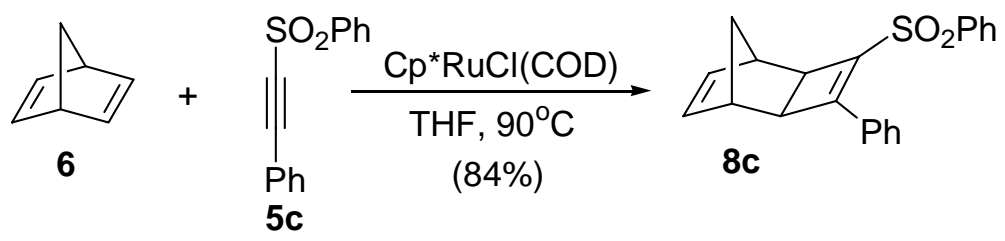

Cycloadduct 8c (Table 1, entry 9). Following the above general procedure (C) with norbornadiene 6 (40.0 $\mu \mathrm{L}, 0.371 \mathrm{mmol})$, alkynyl sulfone 5c (31.2 mg, $0.129 \mathrm{mmol})$, THF (0.3 mL), and Cp*RuCl(COD) (6.6 mg, $0.017 \mathrm{mmol}$ ). The reaction mixture was stirred in the dark at $90{ }^{\circ} \mathrm{C}$ for $72 \mathrm{~h}$. The crude product was purified by column chromatography (EtOAc:hexanes= 1:19) to provide cycloadduct 8c (36.0 mg, $0.1076 \mathrm{mmol}, 84 \%$ ) as a white solid (m.p. $93-95^{\circ} \mathrm{C}$ ). $\mathrm{R}_{\mathrm{f}} 0.52$ (EtOAc:hexanes= 3:7); IR ( $\left.\mathrm{CH}_{2} \mathrm{Cl}_{2}, \mathrm{NaCl}\right) 3063$ (w), 2975 (w), 2949 (w), 1606 (m), 1593 (m), 1489 (w), 1447 (m), 1304 (s), 1150 (s), 1085 (m), 689 (m) cm ${ }^{-1} ;{ }^{1} \mathrm{H}$ NMR $\left(\mathrm{CDCl}_{3}, 400 \mathrm{MHz}\right) \delta$ 7.98-8.03 (m, 4H), 7.61 (m, 1H), 7.51-7.55 
(m, 2H), 7.41-7.48 (m, 3H), 6.16 (dd, 1H, $J=3.0,5.5$ Hz), 6.12 (dd, 1H, $J=3.0,5.5 \mathrm{~Hz}$ ), 2.74 (br s, 1H), 2.71 (d, $1 \mathrm{H}, J=3.8 \mathrm{~Hz}$ ), 2.57 (d, 1H, $J=3.5 \mathrm{~Hz}$ ), 2.48 (br s, $1 \mathrm{H}), 1.28$ (s, $2 \mathrm{H}) ;{ }^{13} \mathrm{C}$ NMR (APT, $\left.\mathrm{CDCl}_{3}, 100 \mathrm{MHz}\right) \delta 155.5,140.9,135.9,135.7,135.3,133.5,130.8,130.7,129.3,129.2,128.7,127.5$, 44.7, 43.7, 39.9, 39.6, 38.8. HRMS calcd. For $\mathrm{C}_{21} \mathrm{H}_{18} \mathrm{SO}_{2}$ : $\mathrm{m} / \mathrm{z}$ 334.1027, found m/z 334.1025. Anal. calcd. For $\mathrm{C}_{21} \mathrm{H}_{18} \mathrm{SO}_{2}$ : C, 75.42; H, 5.42. Found C, 75.52; H, 5.24.

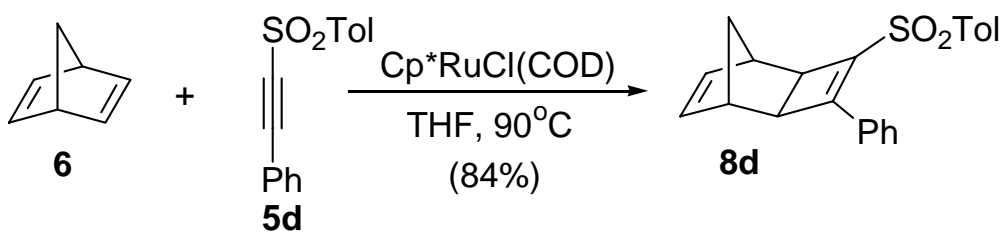

Cycloadduct 8d (Table 1, entry 10). Following the above general procedure (C) with norbornadiene 6 (50.0 $\mu \mathrm{L}, 0.463 \mathrm{mmol})$, alkynyl sulfone 5d (24.9 mg, $0.0971 \mathrm{mmol})$, THF (0.3 mL), and $\mathrm{Cp} * \mathrm{RuCl}(\mathrm{COD})(7.0 \mathrm{mg}, 0.018 \mathrm{mmol})$. The reaction mixture was stirred in the dark at $90{ }^{\circ} \mathrm{C}$ for $72 \mathrm{~h}$. The crude product was purified by column chromatography (EtOAc:hexanes= 1:9) to provide cycloadduct $8 \mathbf{d}$ (28.4 mg, $0.0815 \mathrm{mmol}, 84 \%$ ) as a white solid (m.p. 92 - 95 ${ }^{\circ}$ ). $\quad \mathrm{R}_{\mathrm{f}} \quad 0.43$ (EtOAc:hexanes= 1:4); IR ( $\left.\mathrm{CH}_{2} \mathrm{Cl}_{2}, \mathrm{NaCl}\right) 3061$ (w), 2975 (w), 2948 (w), 2877 (w), 1606 (m), 1595 (m), 1490 (m), 1448 (m), 1312 (m), 1301 (s), 1150 (s), 1085 (m), 718 (m), 686 (m) cm ${ }^{-1}$; ${ }^{1} \mathrm{H}$ NMR $\left(\mathrm{CDCl}_{3}, 400 \mathrm{MHz}\right) \delta$ 7.99-8.02 (m, 2H), 7.85-7.87 (m, 2H), 7.42-7.46 (m, 3H), 7.32 (d, 2H, $J=8.0$ Hz), 6.16 (dd, 1H, $J=3.0,5.4 \mathrm{~Hz}$ ), 6.12 (dd, 1H, $J=3.0,5.4 \mathrm{~Hz}$ ), 2.74 (br s, 1H), 2.70 (d, $1 \mathrm{H}, J=4.0$ $\mathrm{Hz}$ ), 2.56 (d, 1H, $J=3.4 \mathrm{~Hz}$ ), 2.49 (br s, 1H), 2.43 (s, 3H), 1.28 (s, 2H); ${ }^{13} \mathrm{C} \mathrm{NMR}\left(\mathrm{CDCl}_{3}, 100 \mathrm{MHz}\right) \delta$ 154.9, 144.4, 138.0, 135.9, 135.7, 135.7, 130.8, 130.7, 129.8, 129.3, 128.7, 127.6, 44.6, 43.6, 39.9, 39.6, 38.8, 21.6. HRMS calcd. For $\mathrm{C}_{22} \mathrm{H}_{20} \mathrm{SO}_{2}$ : m/z 348.1184, found m/z 348.1190.

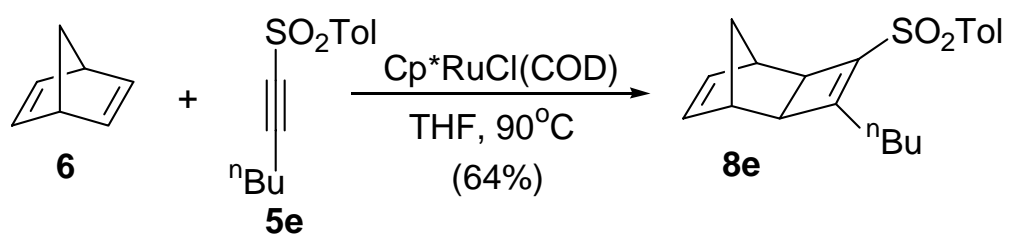

Cycloadduct 8e (Table 1, entry 11). Following the above general procedure (C) with norbornadiene 6 (60.0 $\mu \mathrm{L}, 0.556 \mathrm{mmol})$, alkynyl sulfone 5e (36.0 mg, $0.152 \mathrm{mmol})$, THF (0.3 mL), and Cp*RuCl(COD) (4.7 $\mathrm{mg}, 0.012 \mathrm{mmol}$ ). The reaction mixture was stirred in the dark at $90{ }^{\circ} \mathrm{C}$ for $72 \mathrm{~h}$. The crude product was purified by column chromatography (EtOAc:hexanes=1:9) to provide cycloadduct 8e (32.0 mg, $0.0974 \mathrm{mmol}, 64 \%)$ as a colourless oil. $\mathrm{R}_{\mathrm{f}} 0.48$ (EtOAc:hexanes= 1:4); $\mathrm{IR}\left(\mathrm{CH}_{2} \mathrm{Cl}_{2}, \mathrm{NaCl}\right)$ 3060 (w), 2957 (s), 2873 (m), 1630 (s), 1597 (m), 1455 (m), 1314 (s), 1301 (s), 1291 (s), 1152 (s), 
1127 (m), 1096 (m), 815 (m) cm ${ }^{-1}$; ${ }^{1} \mathrm{H}$ NMR (CDCl, $\left.400 \mathrm{MHz}\right) \delta 7.79$ (d, 2H, J= $\left.8.2 \mathrm{~Hz}\right), 7.32$ (d, $2 \mathrm{H}$, $J=8.0 \mathrm{~Hz}$ ), 6.02-6.07 (m, 2H), 2.44-2.58 (m, 4H), 2.43 (s, 3H), 2.36 (br s, 1H), 2.32 (d, 1H, $J=3.4$ $\mathrm{Hz}), 1.42-1.47$ (m, 2H), 1.31-1.39 (m, 2H), 1.21 (m, 1H), $1.14(\mathrm{~m}, 1 \mathrm{H}), 0.91$ (t, 3H, $J=7.2 \mathrm{~Hz}) ;{ }^{13} \mathrm{C}$ NMR $\left(\mathrm{CDCl}_{3}, 100 \mathrm{MHz}\right) \delta$ 163.9, 144.1, 138.3, 137.4, 135.7, 135.6, 129.8, 127.6, 45.5, 44.7, 39.6, 39.1, 38.4, 28.9, 27.8, 22.8, 21.6, 13.8. HRMS calcd. For $\mathrm{C}_{20} \mathrm{H}_{24} \mathrm{SO}_{2}$ : m/z 328.1497, found m/z 328.1482 .

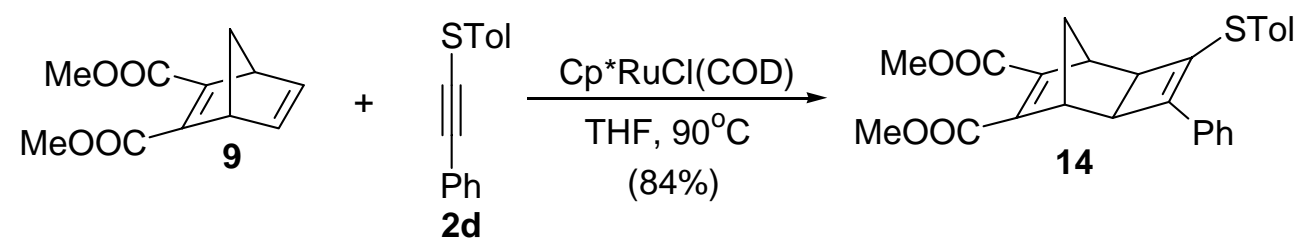

Cycloadduct 14 (Table 2, entry 3). Following the above general procedure (C) with bicyclic alkene 9 (62.0 mg, $0.298 \mathrm{mmol}$ ), alkynyl sulfide 2d (33.4 mg, $0.149 \mathrm{mmol}$ ), THF (0.3 mL), and Cp*RuCl(COD) (5.1 mg, $0.013 \mathrm{mmol})$. The reaction mixture was stirred in the dark at $90{ }^{\circ} \mathrm{C}$ for $168 \mathrm{~h}$. The crude product was purified by column chromatography (EtOAc:hexanes=1:9) to provide cycloadduct 14 (54.1 mg, $0.125 \mathrm{mmol}, 84 \%)$ as a white solid (m.p. $\left.107-108^{\circ} \mathrm{C}\right) . \quad \mathrm{R}_{\mathrm{f}} 0.45$ (EtOAc:hexanes= 3:7); IR ( $\left.\mathrm{CH}_{2} \mathrm{Cl}_{2}, \mathrm{NaCl}\right) 3021$ (m), 2989 (m), 2950 (s), 2876 (w), 1714 (s), 1619 (m), 1604 (m), 1492 (s), 1434 (s), 1331 (s), 1268 (s), 1152 (s), 1087 (s), 1018 (m), 947 (m), 811 (m), 693 (m) cm ${ }^{-1}$; ${ }^{1} \mathrm{H} \mathrm{NMR}_{(\mathrm{CDCl}}$, $400 \mathrm{MHz}) \delta$ 7.51-7.53 (m, 2H), 7.38-7.44 (m, 4H), 7.27 (m, 1H), 7.19 (d, 2H, J = 7.9 Hz), 3.80 (s, 3H), 3.69 (s, 3H), 3.15 (br s, 1H), 3.04 (d, 1H, $J=3.8 \mathrm{~Hz}$ ), 2.79 (d, 1H, $J=3.8 \mathrm{~Hz}$ ), 2.45 (br s, $1 \mathrm{H}), 2.38$ (s, 3H), 1.61-1.65 (m, 2H); ${ }^{13} \mathrm{C} \mathrm{NMR}\left(\mathrm{CDCl}_{3}, 100 \mathrm{MHz}\right) \delta 165.2,164.9,145.1,144.4,139.8,138.5$, 133.7, 133.2, 133.1, 129.8, 128.5, 127.4, 127.3, 125.9, 52.1, 51.8, 46.5, 43.7, 43.3, 43.0, 39.5, 21.2. HRMS calcd. For $\mathrm{C}_{26} \mathrm{H}_{24} \mathrm{SO}_{4}$ : m/z 432.1395, found m/z 432.1391.

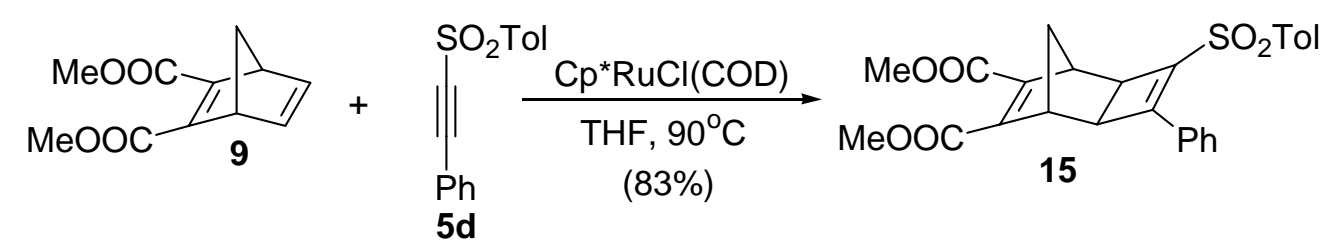

Cycloadduct 15 (Table 2, entry 4). Following the above general procedure (C) with bicyclic alkene 9 (52.0 mg, $0.250 \mathrm{mmol}$ ), alkynyl sulfone 5d (30.1 mg, $0.117 \mathrm{mmol})$, THF (0.3 mL), and $\mathrm{Cp} * \mathrm{RuCl}(\mathrm{COD})$ (6.5 mg, $0.017 \mathrm{mmol})$. The reaction mixture was stirred in the dark at $90{ }^{\circ} \mathrm{C}$ for $72 \mathrm{~h}$. The crude product was purified by column chromatography (EtOAc:hexanes= 3:7) to provide cycloadduct 15 (45.0 mg, 0.0969 mmol, 83\%) as a white solid (m.p. 59 - 62 ${ }^{\circ}$ ). $\mathrm{R}_{\mathrm{f}} 0.11$ 
(EtOAc:hexanes= 1:4); IR ( $\left.\mathrm{CH}_{2} \mathrm{Cl}_{2}, \mathrm{NaCl}\right) 3058$ (w), 2994 (w), 2955 (w), 1717 (s), 1615 (w), 1491 (w), 1436 (w), 1314 (m), 1270 (m), 1152 (s), 1086 (m), 782 (w), 691 (w) cm ${ }^{-1}$; ${ }^{1} \mathrm{H}$ NMR (CDCl, 400 MHz) $\delta$ 8.00-8.02 (m, 2H), 7.86 (d, 2H, $J=8.1 \mathrm{~Hz}), 7.46-7.47$ (m, 3H), 7.34 (d, 2H, $J=8.0 \mathrm{~Hz}), 3.80$ (s, 6H), 3.20 (br s, 1H), 3.04 (d, 1H, J = $3.1 \mathrm{~Hz}$ ), 3.02 (br s, 1H), 2.92 (d, 1H, J = $3.2 \mathrm{~Hz}$ ), 2.43 (s, $3 \mathrm{H}$ ), 1.60 (d, 1H, $J=9.3 \mathrm{~Hz}), 1.49$ (d, $1 \mathrm{H}, J=10.4 \mathrm{~Hz}$ ); ${ }^{13} \mathrm{C}$ NMR (APT, $\left.\mathrm{CDCl}_{3}, 100 \mathrm{MHz}\right) \delta 164.8,164.6$, 153.2, 145.1, 144.8, 143.6, 137.5, 134.2, 131.2, 130.0, 129.4, 128.8, 127.5, 60.3, 52.2, 44.6, 43.4, 43.3, 43.0, 38.9, 21.6, 14.1. HRMS calcd. For $\mathrm{C}_{26} \mathrm{H}_{24} \mathrm{SO}_{6} \mathrm{~m} / \mathrm{z} 464.1294$, found m/z 464.1299.

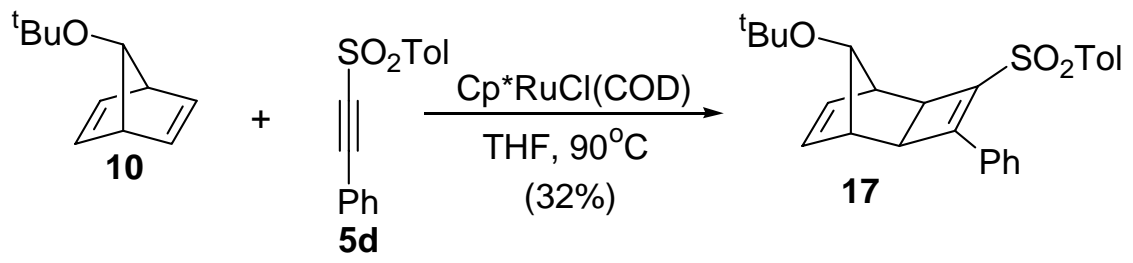

Cycloadduct 17 (Table 2, entry 6). Following the above general procedure (C) with bicyclic alkene 10 (72.2 mg, $0.440 \mathrm{mmol}$ ), alkynyl sulfone 5d (33.9 mg, $0.132 \mathrm{mmol})$, THF (0.3 mL), and $\mathrm{Cp} * \mathrm{RuCl}(\mathrm{COD})(7.1 \mathrm{mg}, 0.019 \mathrm{mmol})$. The reaction mixture was stirred in the dark at $90{ }^{\circ} \mathrm{C}$ for $72 \mathrm{~h}$. The crude product was purified by column chromatography (EtOAc:hexanes= 1:9) to provide cycloadduct 17 (18.0 mg, $0.0428 \mathrm{mmol}, 32 \%)$ as a white solid (m.p. 130 - 131 ${ }^{\circ} \mathrm{C}$ ). $\mathrm{R}_{\mathrm{f}} 0.26$ (EtOAc:hexanes= 1:4); IR ( $\left.\mathrm{CH}_{2} \mathrm{Cl}_{2}, \mathrm{NaCl}\right) 3064$ (w), 2975 (s), 2873 (w), 1596 (m), 1573 (w), 1489 (w), 1448 (w), 1363 (m), 1312 (s), 1301 (s), 1193 (m), 1151 (s), 1108 (m), 1081 (m), 775 (w) cm ; ${ }^{-1} \mathrm{H}$ NMR $\left(\mathrm{CDCl}_{3}, 400 \mathrm{MHz}\right) \delta$ 8.00-8.03 (m, 2H), 7.89 (d, 2H, $\left.J=8.3 \mathrm{~Hz}\right), 7.45-7.47$ (m, 3H), 7.34 (d, 2H, $J=8.0 \mathrm{~Hz}$ ), 6.14 (m, 1H), 6.11 (m, 1H), 3.87 (br s, 1H), 2.73 (br s, 1H), 2.71 (d, 1H, $J=4.3 \mathrm{~Hz}$ ),

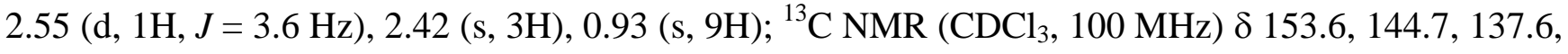
134.3, 132.5, 132.3, 130.8, 130.6, 130.0, 129.2, 128.8, 127.7, 82.5, 73.7, 45.2, 44.0, 43.2, 28.1, 21.6. HRMS calcd. For $\mathrm{C}_{26} \mathrm{H}_{28} \mathrm{SO}_{3}$ : m/z 420.1759, found m/z 420.1763.
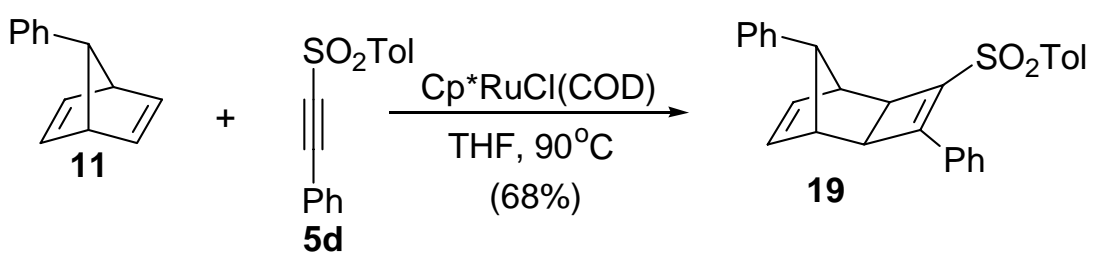

Cycloadduct 19 (Table 2, entry 8). Following the above general procedure (C) with bicyclic alkene 11 (77.1 mg, $0.458 \mathrm{mmol})$, alkynyl sulfone 5d (40.8 mg, $0.159 \mathrm{mmol})$, THF (0.3 mL), and $\mathrm{Cp} * \mathrm{RuCl}(\mathrm{COD})$ (6.4 mg, $0.017 \mathrm{mmol}$ ). The reaction mixture was stirred in the dark at $90{ }^{\circ} \mathrm{C}$ for $72 \mathrm{~h}$. The crude product was purified by column chromatography (EtOAc:hexanes= 1:9) to provide 
cycloadduct 19 (45.9 mg, $0.108 \mathrm{mmol}, 68 \%$ ) as a white solid (m.p. 190 - 193 ${ }^{\circ} \mathrm{C}$ ). $\mathrm{R}_{\mathrm{f}} \quad 0.36$ (EtOAc:hexanes= 1:4); IR ( $\left.\mathrm{CH}_{2} \mathrm{Cl}_{2}, \mathrm{NaCl}\right) 3060$ (w), 2923 (w), 2859 (w), 1740 (w), 1597 (m), 1491

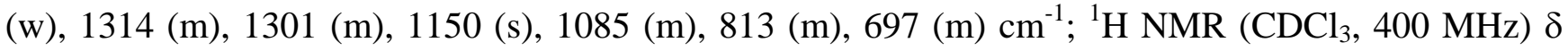
8.09-8.12 (m, 2H), 7.95 (d, 2H, $J=8.3 \mathrm{~Hz}$ ), 7.45-7.51 (m, 3H), 7.35 (d, 2H, $J=8.1 \mathrm{~Hz}), 7.09-7.16$ (m, 3H), 6.75 (d, 2H, $J=7.5 \mathrm{~Hz}$ ), 6.12 (dd, 1H, $J=3.0,2.7 \mathrm{~Hz}$ ), 5.91 (dd, 1H, $J=3.0,2.7 \mathrm{~Hz}$ ), 3.12 (br s, 1H), 2.98 (br s, 1H), 2.89 (d, 1H, $J=3.9 \mathrm{~Hz}$ ), 2.83 (d, 1H, J = $3.9 \mathrm{~Hz}), 2.71$ (br s, $1 \mathrm{H}), 2.44(\mathrm{~s}, 3 \mathrm{H})$; ${ }^{13} \mathrm{C}$ NMR $\left(\mathrm{CDCl}_{3}, 100 \mathrm{MHz}\right) \delta 154.2,144.7,139.5,138.2,134.9,133.0,132.4,130.9,130.6,130.0,129.3$, 128.8, 128.4, 127.7, 127.5, 125.5, 53.3, 45.7, 44.3, 44.1, 43.8, 21.6. HRMS calcd. For $\mathrm{C}_{28} \mathrm{H}_{24} \mathrm{SO}_{2}: \mathrm{m} / \mathrm{z}$ 424.1497, found $\mathrm{m} / \mathrm{z} 424.1490$.

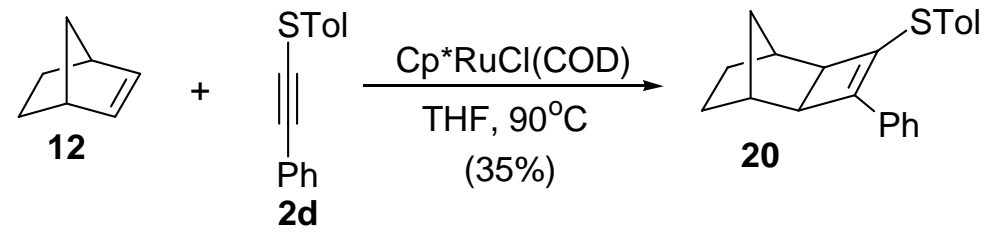

Cycloadduct 20 (Table 2, entry 9). Following the above general procedure (C) with norbornene 12 (47.9 mg, $0.509 \mathrm{mmol}$ ), alkynyl sulfide 2d (36.7 mg, $0.164 \mathrm{mmol}$ ), THF (0.3 mL), and Cp*RuCl(COD) (5.2 mg, $0.014 \mathrm{mmol}$ ). The reaction mixture was stirred in the dark at $90{ }^{\circ} \mathrm{C}$ for $168 \mathrm{~h}$. The crude product was purified by column chromatography (hexanes) to provide cycloadduct 20 (18.4 mg, 0.0578 mmol, 35\%) as a colourless oil. $\mathrm{R}_{\mathrm{f}} 0.57$ (EtOAc:hexanes= 1:9); IR ( $\left.\mathrm{CH}_{2} \mathrm{Cl}_{2}, \mathrm{NaCl}\right) 3020$ (w), 2949 (s), 2868 (m), 1602 (m), 1490 (m), 1446 (m), 1267 (w), 1201 (w), 965 (m), 809 (m), 767 (m), 691 (m) $\mathrm{cm}^{-1}$; ${ }^{1} \mathrm{H}$ NMR $\left(\mathrm{CDCl}_{3}, 400 \mathrm{MHz}\right) \delta$ 7.49-7.51 (m, 2H), 7.34-7.42 (m, 4H), 7.23 (m, 1H), 7.14-7.16 (m, 2H), 2.79 (d, 1H, $J=3.6 \mathrm{~Hz}$ ), 2.59 (d, 1H, $J=3.6 \mathrm{~Hz}$ ), 2.37 (s, 3H), 2.22 (d, 1H, J= $3.8 \mathrm{~Hz}), 1.71$ (d, $1 \mathrm{H}, J=3.5 \mathrm{~Hz}), 1.43-1.59(\mathrm{~m}, 3 \mathrm{H}), 1.10(\mathrm{~m}, 1 \mathrm{H}), 0.95-0.98(\mathrm{~m}, 2 \mathrm{H}) ;{ }^{13} \mathrm{C} \mathrm{NMR}\left(\mathrm{APT}, \mathrm{CDCl}_{3}, 100\right.$ MHz) $\delta 140.8,137.7,134.1,133.0,130.8,129.7,128.4,128.3,126.9,126.0,49.8,46.9$, 35.4, 34.2, 30.7, 28.1, 28.1, 21.2. HRMS calcd. For $\mathrm{C}_{22} \mathrm{H}_{22} \mathrm{~S}$ : $\mathrm{m} / \mathrm{z}$ 318.1442, found m/z 318.1451. Anal. calcd. For $\mathrm{C}_{22} \mathrm{H}_{22} \mathrm{~S}$ : C, 82.97; H, 6.96. Found C, 82.71; H, 7.10.

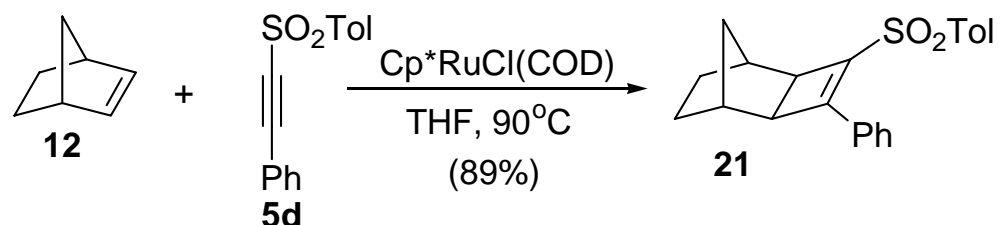

Cycloadduct 21 (Table 2, entry 10). Following the above general procedure (C) with norbornene 12 (47.0 mg, $0.499 \mathrm{mmol})$, alkynyl sulfone 5d (29.5 $\mathrm{mg}, 0.115 \mathrm{mmol})$, THF (0.3 mL), and $\mathrm{Cp} * \mathrm{RuCl}(\mathrm{COD})$ (6.5 mg, $0.017 \mathrm{mmol})$. The reaction mixture was stirred in the dark at $90^{\circ} \mathrm{C}$ for $72 \mathrm{~h}$. 
The crude product was purified by column chromatography (EtOAc:hexanes $=1: 9$ ) to provide cycloadduct 21 (35.9 mg, $0.102 \mathrm{mmol}, 89 \%$ ) as a white solid (m.p. $117-119^{\circ} \mathrm{C}$ ). $\quad \mathrm{R}_{\mathrm{f}} 0.55$ (EtOAc:hexanes= 3:7); IR ( $\left.\mathrm{CH}_{2} \mathrm{Cl}_{2}, \mathrm{NaCl}\right) 3064$ (m), 2958 (s), 2871 (s), 1596 (s), 1490 (s), 1449 (s), 1312 (s), 1150 (s), 1085 (s), 1018 (m), 815 (s), 610 (s) cm ${ }^{-1} ;{ }^{1} \mathrm{H}$ NMR (CDCl $\left.3,400 \mathrm{MHz}\right) \delta$ 7.94-7.97 (m, 2H), 7.84 (d, 2H, $J=8.3 \mathrm{~Hz}$ ), 7.39-7.43 (m, 3H), 7.31 (d, 2H, $J=8.0 \mathrm{~Hz}$ ), 2.81 (d, 1H, $J=3.6 \mathrm{~Hz}$ ), 2.70 (d, 1H, $J=3.6 \mathrm{~Hz}$ ), 2.42 (s, 3H), 2.25 (br s, 1H), 2.07 (d, 1H, $J=2.1 \mathrm{~Hz}$ ), 1.57-1.60 (m, 2H), 1.29 $(\mathrm{d}, 1 \mathrm{H}, J=10.7 \mathrm{~Hz}), 1.11-1.18(\mathrm{~m}, 2 \mathrm{H}), 0.97(\mathrm{~d}, 1 \mathrm{H}, J=10.7 \mathrm{~Hz}) ;{ }^{13} \mathrm{C} \mathrm{NMR}\left(\mathrm{CDCl}_{3}, 100 \mathrm{MHz}\right) \delta$ 152.7, 144.3, 138.1, 132.7, 130.9, 130.4, 129.7, 129.3, 128.6, 127.6, 48.1, 46.8, 35.6, 34.3, 30.4, 28.4, 27.8, 21.6. HRMS calcd. For $\mathrm{C}_{22} \mathrm{H}_{22} \mathrm{SO}_{2}: \mathrm{m} / \mathrm{z}$ 350.1340, found $\mathrm{m} / \mathrm{z} 350.1346$. Anal. calcd. For $\mathrm{C}_{22} \mathrm{H}_{22} \mathrm{SO}_{2}$ : C, 75.39; H, 6.33. Found C, 75.45; H, 6.09.

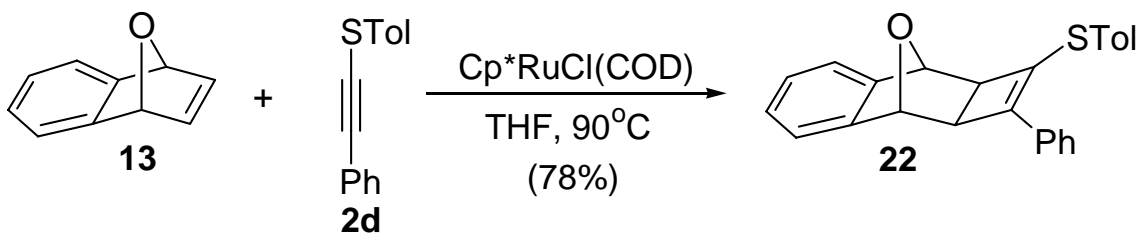

Cycloadduct 22 (Table 2, entry 11). Following the above general procedure (C) with bicyclic alkene 13 (127.0 mg, $0.8809 \mathrm{mmol})$, alkynyl sulfide 2d (42.0 mg, $0.187 \mathrm{mmol})$, THF (0.3 mL), and $\mathrm{Cp} * \mathrm{RuCl}(\mathrm{COD})(5.0 \mathrm{mg}, 0.013 \mathrm{mmol})$. The reaction mixture was stirred in the dark at $90^{\circ} \mathrm{C}$ for $168 \mathrm{~h}$. The crude product was purified by column chromatography (EtOAc:hexanes=1:19) to provide cycloadduct 22 (56.4 mg, $0.153 \mathrm{mmol}, 78 \%$ ) as a white solid (m.p. $112-114^{\circ} \mathrm{C}$ ). $\mathrm{R}_{\mathrm{f}} 0.60$ (EtOAc:hexanes= 3:7); IR ( $\left.\mathrm{CH}_{2} \mathrm{Cl}_{2}, \mathrm{NaCl}\right) 3052$ (w), 3026 (w), 2994 (w), 2949 (w), 1604 (m), 1491 (m), 1458 (m), 1446 (m), 1271 (m), 1194 (m), 1017 (w), 961 (m), 907 (m), 770 (m), 649 (m) cm ${ }^{-1} ;{ }^{1} \mathrm{H}$ NMR ( $\left.\mathrm{CDCl}_{3}, 400 \mathrm{MHz}\right) \delta 7.63$ (d, 2H, $J=7.5 \mathrm{~Hz}$ ), 7.49 (d, 2H, $\left.J=8.0 \mathrm{~Hz}\right), 7.42(\mathrm{t}, 2 \mathrm{H}, J=7.5 \mathrm{~Hz}$ ), 7.28-7.39 (m, 2H), 7.10-7.22 (m, 5H), 5.15 (s, 1H), 4.65 (s, 1H), 3.06 (d, 1H, $J=3.6 \mathrm{~Hz}$ ), 2.82 (d, 1H, $J=3.6 \mathrm{~Hz}$ ), $2.40(\mathrm{~s}, 3 \mathrm{H}) ;{ }^{13} \mathrm{C}$ NMR (APT, $\left.\mathrm{CDCl}_{3}, 100 \mathrm{MHz}\right) \delta 144.9,144.2,141.1,138.1,133.1$, 132.8, 131.2, 129.9, 128.5, 128.3, 127.6, 126.6, 126.6, 126.0, 119.7, 119.6, 76.4, 75.9, 48.4, 45.3, 21.2. HRMS calcd. For $\mathrm{C}_{25} \mathrm{H}_{20} \mathrm{SO}: \mathrm{m} / \mathrm{z}$ 368.1235, found m/z 368.1243.

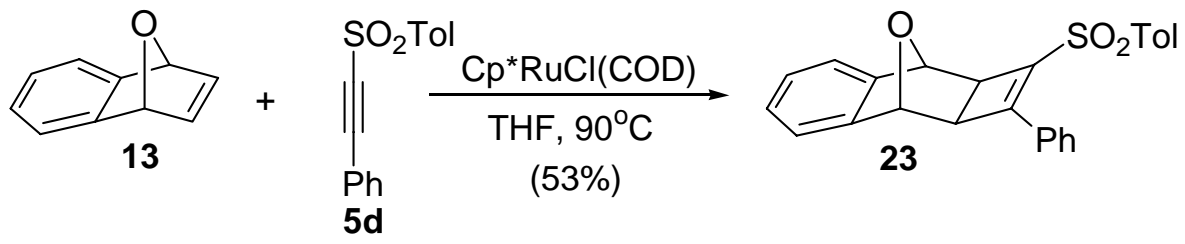

Cycloadduct 23 (Table 2, entry 12). Following the above general procedure (C) with bicyclic alkene 13 (62.0 mg, $0.430 \mathrm{mmol}$ ), alkynyl sulfone 5 d (34.5 mg, $0.135 \mathrm{mmol})$, THF (0.3 mL), and 
Cp*RuCl(COD) $(7.6 \mathrm{mg}, 0.020 \mathrm{mmol})$. The reaction mixture was stirred in the dark at $90{ }^{\circ} \mathrm{C}$ for $72 \mathrm{~h}$. The crude product was purified by column chromatography (EtOAc:hexanes= 1:4) to provide cycloadduct 23 (28.4 mg, $0.0709 \mathrm{mmol}, 53 \%$ ) as a white solid (m.p. $164-165^{\circ} \mathrm{C}$ ). $\quad \mathrm{R}_{\mathrm{f}} \quad 0.50$ (EtOAc:hexanes= 2:3); IR ( $\left.\mathrm{CH}_{2} \mathrm{Cl}_{2}, \mathrm{NaCl}\right) 3058$ (w), 3032 (w), 3000 (w), 2961 (w), 1608 (m), 1596 (m), 1491 (m), 1459 (w), 1312 (m), 1301 (m), 1149 (s), 1083 (m), 907 (w), 697 (m) cm ${ }^{-1}{ }^{1} \mathrm{H}$ NMR $\left(\mathrm{CDCl}_{3}, 400 \mathrm{MHz}\right) \delta$ 7.99-8.02 (m, 2H), 7.93 (d, 2H, $\left.J=8.3 \mathrm{~Hz}\right), 7.44-7.48$ (m, 3H), 7.28-7.34 (m, 4H), 7.19-7.22 (m, 2H), 5.17 (s, 1H), 4.91 (s, 1H), 3.06 (d, 1H, J = 3.6 Hz), 3.01 (d, 1H, J = 3.6 Hz), 2.41 (s, 3H); ${ }^{13} \mathrm{C}$ NMR (APT, $\left.\mathrm{CDCl}_{3}, 100 \mathrm{MHz}\right) \delta$ 152.7, 144.7, 143.8, 143.6, 138.0, 133.2, 131.1, 130.3, 129.9, 129.2, 128.8, 127.4, 127.2, 127.1, 120.2, 119.7, 76.4, 75.6, 46.4, 45.3, 21.7. HRMS calcd. For $\mathrm{C}_{25} \mathrm{H}_{20} \mathrm{SO}_{3} \mathrm{~m} / \mathrm{z} 400.1133$, found $\mathrm{m} / \mathrm{z} 400.1140$.

\section{Part III: Conversion of Cycloadduct 8c to Cycloadducts 25a-e}

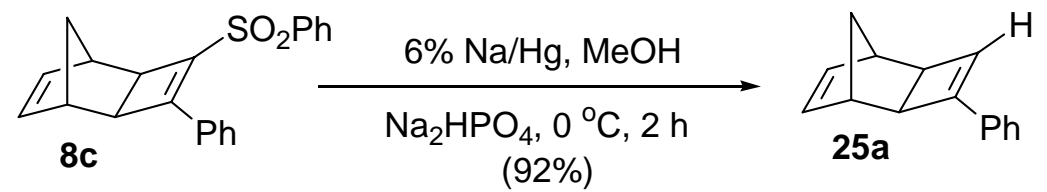

Cycloadduct 25a (Scheme 2). $6 \% \mathrm{Na} / \mathrm{Hg}$ (243.4 mg, $0.6352 \mathrm{mmol}, 9 \mathrm{eq})$ was added to a stirred solution of cycloadduct 8c (23.6 mg, $0.0677 \mathrm{mmol}, 1 \mathrm{eq}), \mathrm{Na}_{2} \mathrm{HPO}_{4}$ (61.8 mg, $\left.0.4353 \mathrm{mmol}, 6 \mathrm{eq}\right)$, and $\mathrm{MeOH}(5 \mathrm{~mL})$ under nitrogen at $0^{\circ} \mathrm{C}$. The reaction mixture was allowed to stir at $0^{\circ} \mathrm{C}$ for $2 \mathrm{~h}$. The reaction mixture was then diluted with ethyl acetate and filtered through a plug of silica. The filtrate was evaporated to give the crude product, which was purified by column chromatography (hexanes) to provide cycloadduct 25a (12.1 mg, $0.0623 \mathrm{mmol}, 92 \%)$ as a colourless oil. $\mathrm{R}_{\mathrm{f}} 0.64$ (EtOAc:hexanes= 1:19); ${ }^{1} \mathrm{H} \mathrm{NMR}\left(\mathrm{CDCl}_{3}, 400 \mathrm{MHz}\right) \delta$ 7.34-7.40 (m, 2H), 7.30 (t, 2H, J = 7.3 Hz), 7.18 (m, 1H), 6.48 (s, 1H), 6.13-6.17 (m, 2H), 2.69 (d, 1H, $J=3.2$ Hz), 2.61 (br s, 1H), 2.45 (br s, 1H), 2.35 (d, 1H, $J=3.4$ $\mathrm{Hz}$ ), 1.46 (d, $1 \mathrm{H}, J=9.0 \mathrm{~Hz}), 1.27$ (d, $1 \mathrm{H}, J=9.0 \mathrm{~Hz}) ;{ }^{13} \mathrm{C} \mathrm{NMR}\left(\mathrm{CDCl}_{3}, 100 \mathrm{MHz}\right) \delta 148.6,136.4$, 135.4, 133.9, 129.4, 128.4, 127.6, 124.8, 45.0, 42.4, 40.0, 39.3, 38.2. The spectral data are identical to those reported in the literature. ${ }^{17}$

${ }^{17}$ (a) U. Azzena, S. Cossu, O. D. Lucchi, G. Licini, L. Pasquatos, G. Valle Gazz. Chim. Ital. 1990, 120, 557. (b) S. Cossu, O. D. Lucchi Gazz. Chim. Ital. 1990, 120, 569. 


\section{General Procedure (D) for the Reaction between Sulfonyl Containing Cycloadduct 8c with}

Organolithium Reagents. In an oven-dried vial, the cycloadduct 8c (1 eq) was dissolved in THF $(\sim 0.4 \mathrm{M})$ under nitrogen, and cooled to $0^{\circ} \mathrm{C}$. The organolithium reagent (3 eq) was then added slowly via syringe and the resulting reaction mixture was stirred at $25^{\circ} \mathrm{C}$. The reaction was quenched with a drop of water and the crude product was purified by flash chromatography (hexanes or ethyl acetate/hexanes mixture).

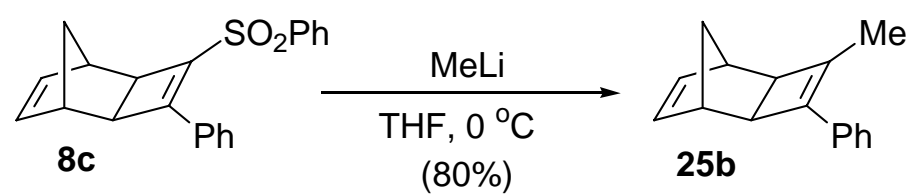

Cycloadduct 25b (Table 4, entry 1). Following the above general procedure (D) with cycloadduct 8c (23.9 mg, $0.0715 \mathrm{mmol}$ ), MeLi (1.4 M, $0.150 \mathrm{~mL}, 0.210 \mathrm{mmol}$ ), and THF (0.2 mL). The crude product was purified by column chromatography (EtOAc:hexanes= 1:9) to provide cycloadduct 25b (11.9 mg, $0.0571 \mathrm{mmol}, 80 \%)$ as a colourless oil. $\mathrm{R}_{\mathrm{f}} 0.74$ (EtOAc:hexanes= 3:7); ${ }^{1} \mathrm{H} \mathrm{NMR}\left(\mathrm{CDCl}_{3}, 400 \mathrm{MHz}\right) \delta$ 7.33-7.42 (m, 4H), 7.21 (m, 1H), 6.14-6.19 (m, 2H), 2.66 (br s, 1H), 2.56 (br s, 1H), 2.53 (br s, 1H), 2.27 (d, 1H, $J=2.5 \mathrm{~Hz}), 2.02$ (s, 3H), 1.38 (d, $1 \mathrm{H}, J=8.9 \mathrm{~Hz}), 1.28$ (d, $1 \mathrm{H}, J=8.9 \mathrm{~Hz}) ;{ }^{13} \mathrm{C}$ NMR (APT, $\left.\mathrm{CDCl}_{3}, 100 \mathrm{MHz}\right) \delta$ 141.3, 139.7, 135.9, 135.7, 135.3, 128.4, 126.5, 125.7, 45.7, 42.6, 40.0, 39.2, 38.1, 14.2. The spectral data are identical to those reported in the literature. ${ }^{18}$

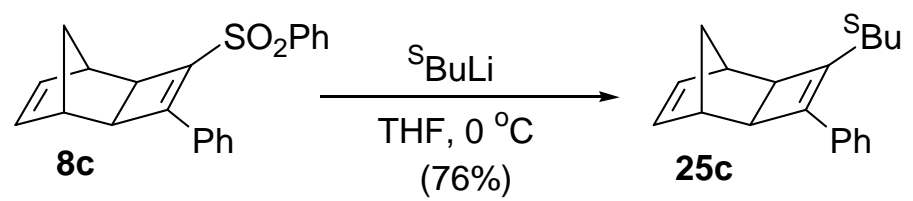

Cycloadduct 25c (Table 4, entry 2). Following the above general procedure (D) with cycloadduct 8c (19.4 mg, $0.0580 \mathrm{mmol})$, sBuLi (1.0 M, $0.200 \mathrm{~mL}, 0.200 \mathrm{mmol})$, and THF (0.2 mL). The crude product was purified by column chromatography (hexanes) to provide cycloadduct 25c (11.0 mg, 0.0439 mmol, 76\%) as a colourless oil. $\mathrm{R}_{\mathrm{f}} 0.74$ (EtOAc:hexanes= 3:7); IR $\left(\mathrm{CH}_{2} \mathrm{Cl}_{2}, \mathrm{NaCl}\right) 3079$ (w), 3056 (m), 2963 (s), 2926 (s), 2872 (m), 1639 (w), 1598 (w), 1493 (m), 1446 (m), 1321 (m), 1175 (w), 1056 (w), $716(\mathrm{~m}) \mathrm{cm}^{-1} ;{ }^{1} \mathrm{H} \mathrm{NMR}\left(\mathrm{CDCl}_{3}, 400 \mathrm{MHz}\right) \delta 7.42$ (d, 2H, $\left.J=7.4 \mathrm{~Hz}\right), 7.34$ (t, 2H, $J=7.7$ Hz), 7.20 (t, 1H, J = 7.4 Hz), 6.13-6.18 (m, 2H), 2.85 (m, 1H), 2.65 (m, 1H), 2.53-2.67 (m, 1.5H), 2.50 (d, 0.5H, $J=3.7 \mathrm{~Hz}), 3.35$ (d, 0.5H, $J=5.5 \mathrm{~Hz}$ ), 2.33 (d, 0.5H, $J=4.3 \mathrm{~Hz}), 1.38-1.66$ (m, 3H), 1.29 (m, 1H), 1.14 (d, 1.5H, $J=6.9 \mathrm{~Hz}), 1.10$ (d, 1.5H, $J=6.8 \mathrm{~Hz}), 0.99(\mathrm{t}, 1.5 \mathrm{H}, J=7.4 \mathrm{~Hz}), 0.91(\mathrm{t}, 1.5 \mathrm{H}, J=$

\footnotetext{
${ }^{18}$ Villeneuve, K.; Riddell, N.; Jordan, R.W.; Tsui, G.C.; Tam, W. Organic Letters 2004, 6, 4543.
} 
$7.4 \mathrm{~Hz}) ;{ }^{13} \mathrm{C} \mathrm{NMR}\left(\mathrm{CDCl}_{3}, 75 \mathrm{MHz}\right) \delta 150.1,149.7,138.8,138.1,135.8,135.7,135.6,135.3,135.3$, 128.4, 126.5, 126.1, 126.1, 42.0, 41.9, 41.7, 40.1, 40.0, 40.0, 39.9, 39.1, 39.0, 35.9, 35.8, 28.6, 28.4, 18.9, 18.2, 12.8, 12.3. HRMS calcd. For $\mathrm{C}_{19} \mathrm{H}_{22}: \mathrm{m} / \mathrm{z} 250.1721$, found $\mathrm{m} / \mathrm{z} 250.1724$.

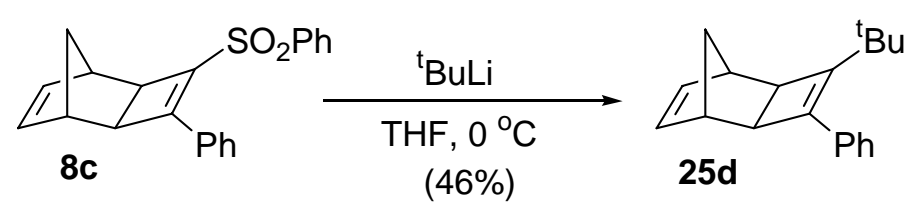

Cycloadduct 25d (Table 4, entry 3). Following the above general procedure (D) with cycloadduct 8c (19.5 mg, $0.0583 \mathrm{mmol})$, tBuLi (1.7 M, $0.120 \mathrm{~mL}, 0.204 \mathrm{mmol})$, and THF $(0.2 \mathrm{~mL})$. The crude product was purified by column chromatography (EtOAc:hexanes=1:49) to provide cycloadduct $25 \mathbf{d}$ (6.7 mg, $0.027 \mathrm{mmol}, 46 \%$ ) as a white solid (m.p. $45-46^{\circ} \mathrm{C}$ ). $\mathrm{R}_{\mathrm{f}} 0.74$ (EtOAc:hexanes= 3:7); IR ( $\left.\mathrm{CH}_{2} \mathrm{Cl}_{2}, \mathrm{NaCl}\right) 3057$ (w), 2962 (s), 2866 (m), 1597 (m), 1490 (w), 1446 (w), 1362 (m), 1321 (m), 1214 (m), 967 (w), $776(\mathrm{~m}) \mathrm{cm}^{-1}$; ${ }^{1} \mathrm{H}$ NMR (CDCl, $\left.400 \mathrm{MHz}\right) \delta$ 7.30-7.38 (m, 4H), $7.21(\mathrm{~m}, 1 \mathrm{H}), 6.12-6.16$ (m, 2H), 2.60 (br s, 1H), 2.51 (br s, 1H), 2.44 (d, 1H, $J=3.7 \mathrm{~Hz}$ ), 2.31 (d, 1H, $J=3.7 \mathrm{~Hz}$ ), 1.27 (d, 2H, $J=9.6 \mathrm{~Hz}), 1.18(\mathrm{~s}, 9 \mathrm{H}) ;{ }^{13} \mathrm{C} \mathrm{NMR}\left(\mathrm{CDCl}_{3}, 100 \mathrm{MHz}\right) \delta 152.7,138.7,136.4,135.7,135.6,127.9$, 127.8, 126.5, 42.7, 42.4, 40.0, 39.6, 39.0, 33.8, 29.1. HRMS calcd. For $\mathrm{C}_{19} \mathrm{H}_{22}: \mathrm{m} / \mathrm{z} 250.1721$, found $\mathrm{m} / \mathrm{z} 250.1729$.

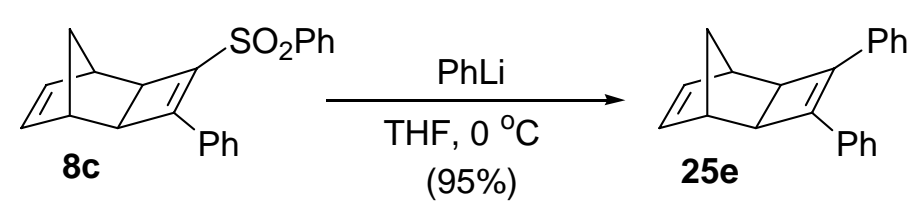

Cycloadduct 25e (Table 4, entry 4). Following the above general procedure (D) with cycloadduct 8c (18.1 mg, $0.0541 \mathrm{mmol}$ ), PhLi (2.0 M, $0.150 \mathrm{~mL}, 0.300 \mathrm{mmol})$, and THF (0.2 mL). The crude product was purified by column chromatography (EtOAc:hexanes= 1:49) to provide cycloadduct $25 \mathbf{e}(13.9 \mathrm{mg}$, 0.0514 mmol, 95\%) as a white solid. $\mathrm{R}_{\mathrm{f}} 0.72$ (EtOAc:hexanes= 3:7); ${ }^{1} \mathrm{H} \mathrm{NMR}\left(\mathrm{CDCl}_{3}, 400 \mathrm{MHz}\right) \delta$ 7.57-7.60 (m, 4H), 7.32-7.36 (m, 4H), 7.24-7.28 (m, 2H), 6.23 (s, 2H), 2.73 (br s, 2H), 2.68 (s, 2H), 1.58 (d, $1 \mathrm{H}, J=9.1 \mathrm{~Hz}), 1.35$ (d, $1 \mathrm{H}, J=9.0 \mathrm{~Hz}) ;{ }^{13} \mathrm{C} \mathrm{NMR}\left(\mathrm{CDCl}_{3}, 100 \mathrm{MHz}\right) \delta 141.1,135.9,135.3$, 128.4, 127.6, 126.3, 43.5, 40.0, 39.5. The spectral data are identical to those reported in the literature. ${ }^{18}$ 